DOI 10.4171/JEMS/399

Jean Bourgain $\cdot$ Nicolas Burq $\cdot$ Maciej Zworski

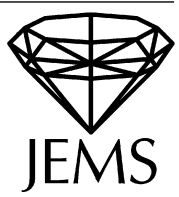

\title{
Control for Schrödinger operators on 2-tori: rough potentials
}

Received January 6, 2013

Abstract. For the Schrödinger equation $\left(i \partial_{t}+\Delta\right) u=0$ on a torus, an arbitrary non-empty open set $\Omega$ provides control and observability of the solution: $\left\|\left.u\right|_{t=0}\right\|_{L^{2}\left(\mathbb{T}^{2}\right)} \leq K_{T}\|u\|_{L^{2}((0, T) \times \Omega)}$. We show that the same result remains true for $\left(i \partial_{t}+\Delta-V\right) u=0$ where $V \in L^{2}\left(\mathbb{T}^{2}\right)$, and $\mathbb{T}^{2}$ is a (rational or irrational) torus. That extends the results of [1], and [8] where the observability was proved for $V \in C\left(\mathbb{T}^{2}\right)$ and conjectured for $V \in L^{\infty}\left(\mathbb{T}^{2}\right)$. The higher dimensional generalization remains open for $V \in L^{\infty}\left(\mathbb{T}^{n}\right)$.

\section{Introduction}

The purpose of this paper is to prove a case of the conjecture made by the last two authors in [8]. It concerned control and observability for Schrödinger operators on tori with $L^{\infty}$ potentials. Here we prove that for two-dimensional tori the desired results are valid for potentials which are merely in $L^{2}$.

To state the result consider

$$
\begin{gathered}
\mathbb{T}^{2}:=\mathbb{R}^{2} / A \mathbb{Z} \times B \mathbb{Z}, \quad A, B \in \mathbb{R} \backslash\{0\}, \quad V \in L^{2}\left(\mathbb{T}^{2}\right), \\
(-\Delta+V(z)-\lambda) u(z)=f(z), \quad z \in \mathbb{T}^{2},
\end{gathered}
$$

and

$$
i \partial_{t} u(t, z)=(-\Delta+V(z)) u(t, z), \quad z \in \mathbb{T}^{2},
$$

The first theorem concerns solutions of the stationary Schrödinger equation and is applicable to high energy eigenfunctions:

J. Bourgain: School of Mathematics, Institute for Advanced Study, Princeton, NJ 08540, USA; e-mail: bourgain@math.ias.edu

N. Burq: Université Paris Sud, Mathématiques, Bât. 425, 91405 Orsay Cedex, France, and École Normale Supérieure, 45, rue d'Ulm, 75005 Paris, Cedex 05, France; e-mail: nicolas.burq@math.u-psud.fr

M. Zworski: Mathematics Department, University of California, Berkeley, CA 94720, USA; e-mail: zworski@math.berkeley.edu 
Theorem 1. Let $\Omega \subset \mathbb{T}^{2}$ be a non-empty open set. There exists a constant $K=K(\Omega)$, depending only on $\Omega$, such that for any solution of (1.1) we have

$$
\|u\|_{L^{2}\left(\mathbb{T}^{2}\right)} \leq K\left(\|f\|_{L^{2}\left(\mathbb{T}^{2}\right)}+\|u\|_{L^{2}(\Omega)}\right) .
$$

Theorem 1 can be deduced from the following dynamical result:

Theorem 2. Let $\Omega \subset \mathbb{T}^{2}$ be a non-empty open set and let $T>0$. There exists a constant $K$, depending only on $\Omega, T$ and $V$, such that for any solution of (1.2) we have

$$
\|u(0, \cdot)\|_{L^{2}\left(\mathbb{T}^{2}\right)}^{2} \leq K \int_{0}^{T}\|u(t, \cdot)\|_{L^{2}(\Omega)}^{2} d t .
$$

An estimate of this type is called an observability result. Once we have it, the HUM method (see [19]) automatically provides the following control result:

Theorem 3. Let $\Omega \subset \mathbb{T}^{2}$ be any non-empty open set and let $T>0$. For any $u_{0} \in L^{2}\left(\mathbb{T}^{2}\right)$, there exists $f \in L^{2}([0, T] \times \Omega)$ such that the solution of the equation

$$
\left(i \partial_{t}+\Delta-V(z)\right) u(t, z)=f \mathbb{1}_{[0, T] \times \Omega}(t, z), \quad u(0, \cdot)=u_{0},
$$

satisfies

$$
u(T, \cdot) \equiv 0 .
$$

In the case of $V \equiv 0$ (and rational tori) the estimates (1.3) and (1.4) were proved by Jaffard [13] and Haraux [12] (in dimension 2) and Komornik [16] (in higher dimensions) using Kahane's work [17] on lacunary Fourier series. For $V \in C^{\infty}\left(\mathbb{T}^{2}\right)$ the results above were proved by the last two authors [8], and for a class potentials including continuous potentials on $\mathbb{T}^{n}$, by Anantharaman-Macia [1]. The paper [1] resolves other questions concerning semiclassical measures on tori and contains further references; see also [4]. For a presentation of other aspects of control theory for the Schrödinger equation we refer to [18]—see also [6, §3].

The paper is organized as follows. In $\S 2$ we present dispersive estimates which allow approximation of rough potentials by smooth potentials. In $\$ 3$ we refine some of the onedimensional observability estimates and show that they hold for potentials $W \in L^{p}\left(\mathbb{T}^{1}\right)$, $p>1$. The next $\S 4$ is devoted to semiclassical observability estimates for a family of smooth potentials compact in $L^{2}\left(\mathbb{T}^{2}\right)$. In the following section an observability result is proved for general tori with constants uniform in a compact set in $L^{2}$ (Proposition 5.1(i)). Combined with the results from $\S 2$, that gives the proof of the theorem.

\section{A priori estimates for solutions to Schrödinger equations}

The proof of observability for rough potentials will follow from observability for smooth potentials with estimates controlled by constants depending only on $L^{2}$ norms of the potential. The approximation argument uses dispersion estimates for the Schrödinger group on the torus and we first show that these estimates hold in the presence of a potential. 


\subsection{The case of $\mathbb{T}^{1}$}

We start with the simpler case of one-dimensional equations. It will be needed in $\S 3$ but it also introduces the idea of the proof in an elementary setting.

We first make some general comments. The operator $-\partial_{x}^{2}+W, W \in L^{1}\left(\mathbb{T}^{1}\right)$, is defined by Friedrichs' extension (see for instance [10, Theorem 4.10]) using the quadratic form

$$
q(v, v)=\int_{\mathbb{T}^{1}}\left(\left|\partial_{x} v(x)\right|^{2}+W(x)|v(x)|^{2}\right) d x, \quad v \in H^{1}\left(\mathbb{T}^{1}\right),
$$

which is bounded from below since

$$
\begin{aligned}
\left.\left|\int_{\mathbb{T}^{1}} W(x)\right| v(x)\right|^{2} d x \mid & \leq C\|W\|_{L^{1}}\|u\|_{L^{\infty}}^{2} \leq C\|W\|_{L^{1}}\left\|\partial_{x} v\right\|_{L^{2}}\|v\|_{L^{2}} \\
& \leq-C \epsilon\|W\|_{L^{1}}\left\|\partial_{x} v\right\|_{L^{2}}^{2}-\frac{C}{\epsilon}\|W\|_{L^{1}}\|v\|_{L^{2}}^{2} .
\end{aligned}
$$

Hence $P=-\partial_{x}^{2}+W$ defined on $C^{\infty}\left(\mathbb{T}^{1}\right)$ has a unique self-adjoint extension with the domain containing $H^{1}\left(\mathbb{T}^{1}\right)$. When $W \in L^{2}\left(\mathbb{T}^{1}\right)$ the operator is self-adjoint with the domain $H^{2}\left(\mathbb{T}^{1}\right)$. The resolvent, $\left(-\partial_{x}^{2}+W-z\right)^{-1}, z \notin \mathbb{R}$, is compact and the spectrum is discrete with eigenvalues $\lambda_{j} \rightarrow \infty$.

The following estimate applies to solutions of the Schrödinger equation satisfying the Floquet periodicity conditions

$$
v(x+2 \pi)=e^{2 \pi i k} v(x),
$$

or equivalently to solutions of the Schrödinger equation with $\partial_{x}$ replaced by $\partial_{x}+i k$. (We note that $u(x):=e^{-i k x} v(x)$ is periodic and $\partial_{x} v(x)=e^{i k x}\left(\partial_{x}+i k\right) u(x)$.)

Proposition 2.1. For any $W \in L^{2}\left(\mathbb{T}^{1}\right)$, there exists $C>0$ such that for any $k \in[0,1)$ and $u_{0} \in L^{2}\left(\mathbb{T}^{1}\right)$, the solution to the Schrödinger equation

$$
\left(i \partial_{t}+\left(\partial_{x}+i k\right)^{2}-W\right) u=0,\left.\quad v\right|_{t=0}=u_{0},
$$

satisfies

$$
\|u\|_{L^{\infty}\left(\mathbb{T}_{x}^{1} ; L^{2}(0, T)\right)} \leq C(1+\sqrt{T})\left(1+\|W\|_{L^{2}\left(\mathbb{T}^{1}\right)}\right)\left\|u_{0}\right\|_{L^{2}\left(\mathbb{T}^{1}\right)} .
$$

Proof. For $W \equiv 0$ we put $T=2 \pi$ so that, with $c_{n}=\hat{u}_{0}(n)$, we have

$$
\begin{aligned}
\left\|e^{i t \partial_{x}^{2}} u_{0}\right\|_{L_{x}^{\infty} L_{t}^{2}}^{2} & =\sup _{x} \int_{0}^{2 \pi}\left|\sum_{n \in \mathbb{Z}} c_{n} e^{-i t|n+k|^{2}+i n x}\right|^{2} d t \\
& =\sup _{x} \sum_{n, m \in \mathbb{Z}} \int_{0}^{2 \pi} e^{i\left(|n+k|^{2}-|m+k|^{2}\right) t} e^{i(n-m) x} c_{n} \bar{c}_{m} d t \\
& =\sup _{x} \sum_{n \in \mathbb{Z}}\left|\sum_{\substack{m \in \mathbb{Z} \\
\pm(m+k)=n+k}} c_{m} e^{i m x}\right|^{2} \leq 4 \sum_{n \in \mathbb{Z}}\left|c_{n}\right|^{2} \leq C\left\|u_{0}\right\|_{L^{2}\left(\mathbb{T}^{1}\right)}^{2} .
\end{aligned}
$$


(We note that $\pm(m+k)=n+k$ has one solution only when $k \neq 0,1 / 2$ and two solutions $m= \pm n$ for $k=0$ and $m=n,-n-1$ for $k=1 / 2$.) For a non-zero potential $W \in L^{2}\left(\mathbb{T}^{1}\right)$ we use Duhamel's formula to write

$$
u(t)=e^{i t \partial_{x}^{2}} u_{0}+\frac{1}{i} \int_{0}^{t} e^{i(t-s) \partial_{x}^{2}}(W u(s)) d s .
$$

Applying (2.4) (now with a small $T>0$ ) and the Minkowski inequality we obtain

$$
\begin{aligned}
\|u\|_{L_{x}^{\infty} L_{t}^{2}(0, T)} & \leq C\left\|u_{0}\right\|_{L_{x}^{2}}+\int_{0}^{T}\left\|\mathbb{1}_{s<t} e^{i(t-s) \Delta}(W u(s))\right\|_{L_{x}^{\infty} L_{s}^{2}(0, T)} d s \\
& \leq C\left\|u_{0}\right\|_{L_{x}^{2}}+\int_{0}^{T}\left\|e^{i(t-s) \Delta}(W u(s))\right\|_{L_{x}^{\infty} L_{s}^{2}(0, T)} d s \\
& \leq C\left\|u_{0}\right\|_{L_{x}^{2}}+C \int_{0}^{T}\|W u(s)\|_{L_{x}^{2}} d s \\
& \leq C\left\|u_{0}\right\|_{L_{x}^{2}}+C \sqrt{T}\|W\|_{L^{2}}\|u\|_{L_{x}^{\infty} L_{t}^{2}(0, T)} .
\end{aligned}
$$

Hence

$$
\|u\|_{L_{x}^{\infty} L_{t}^{2}(0, T)} \leq 2 C\|u\|_{L_{x}^{2}} \quad \text { if } \sqrt{T}\|W\|_{L^{2}} \leq 1 / 4 .
$$

To obtain the estimate for multiples of $T$ satisfying (2.6) we note that, by the invariance of the $L_{x}^{2}$ norm of $u(t), \int_{(k-1) T}^{k T}\|u(t)\|_{L_{x}^{\infty}}^{2} d t \leq 2 C\|u((k-1) t)\|_{L_{x}^{2}}=2 C\left\|u_{0}\right\|_{L_{x}^{2}}$. Iterating this inequality gives (2.3).

\subsection{The case of two-dimensional tori}

We now assume that $A=2 \pi, B=2 \pi \gamma^{-1}>0$ in the definition of $\mathbb{T}^{2}$. The case of general $A, B$ follows by rescaling. For $n=\left(n_{1}, n_{2}\right) \in \mathbb{Z}^{2}$, we set

$$
|n|=\sqrt{n_{1}^{2}+\gamma n_{2}^{2}}, \quad n \cdot x=n_{1} x_{1}+\gamma n_{2} x_{2} .
$$

We start with some general observations. If $V \in L^{2}\left(\mathbb{T}^{2} ; \mathbb{R}\right)$ then $-\Delta+V$ on $C^{\infty}\left(\mathbb{T}^{2}\right)$ is a symmetric operator. Also, by Sobolev inequalities,

$$
(-\Delta+i)^{-1}: L^{2}\left(\mathbb{T}^{2}\right) \rightarrow H^{2}\left(\mathbb{T}^{2}\right) \hookrightarrow C^{1-\varepsilon}\left(\mathbb{T}^{2}\right) \hookrightarrow L^{\infty}\left(\mathbb{T}^{2}\right),
$$

is a compact operator. Hence, as multiplication by $V \in L^{2}$ is bounded $L^{\infty} \rightarrow L^{2}$, $V(-\Delta+i)^{-1}$ is a compact operator on $L^{2}$. It follows that the operator $-\Delta+V$ is essentially self-adjoint and has a discrete spectrum (see for instance [10, Theorem 4.19]). Since for $u \in H^{2}\left(\mathbb{T}^{2}\right) \subset L^{\infty}\left(\mathbb{T}^{2}\right)$, we have $V u \in L^{2}$, the domain is equal to $H^{2}\left(\mathbb{T}^{2}\right)$. In particular,

$$
u(t):=e^{i t(\Delta-V)} u_{0} \in C^{0}\left(\mathbb{R}_{t} ; H^{2}\left(\mathbb{T}^{2}\right)\right) \cap C^{1}\left(\mathbb{R}_{t} ; L^{2}\left(\mathbb{T}^{2}\right)\right)
$$

and

$$
u(t)=e^{i t \Delta} u_{0}+\frac{1}{i} \int_{0}^{t} e^{i(t-s) \Delta}(V u(s)) d s .
$$


Proposition 2.2. Let $T>0$. For any compact subset $\mathcal{V} \subset L^{2}\left(\mathbb{T}^{2}\right)$, there exist $C(\mathcal{V})$ and $\epsilon>0$ such that for any $V \in \mathcal{V}+B(0, \epsilon) \subset L^{2}\left(\mathbb{T}^{2}\right)$ and any

$$
v_{0} \in L^{2}\left(\mathbb{T}^{2}\right), \quad f \in L^{1}\left((0, T) ; L^{2}\left(\mathbb{T}^{2}\right)\right)+L^{4 / 3}\left(\mathbb{T}^{2} ; L^{2}(0, T)\right),
$$

the solution to

$$
\left(i \partial_{t}+(\Delta-V)\right) u=f,\left.\quad u\right|_{t=0}=v_{0},
$$

satisfies

$$
\begin{aligned}
\|u\|_{L^{\infty}\left((0, T) ; L^{2}\left(\mathbb{T}^{2}\right)\right) \cap L^{4}\left(\mathbb{T}_{x}^{2} ; L^{2}(0, T)\right)} & \\
& \leq C(\mathcal{V})\left(\left\|v_{0}\right\|_{L^{2}\left(\mathbb{T}^{2}\right)}+\|f\|_{L^{1}\left((0, T) ; L^{2}\left(\mathbb{T}^{2}\right)\right)+L^{4 / 3}\left(\mathbb{T}^{2} ; L^{2}(0, T)\right)}\right) .
\end{aligned}
$$

Before proving this result, let us show how it implies that Jaffard's result (Theorem 2 with $V=0)$ is stable under perturbation with potentials small in $L^{2}\left(\mathbb{T}^{2}\right)$ :

Corollary 2.3. For any non-empty open set $\Omega$ and $T>0$, there exist constants $\kappa, K>0$ such that for $V \in L^{2}\left(\mathbb{T}^{2}\right)$,

$$
\|V\|_{L^{2}\left(\mathbb{T}^{2}\right)} \leq \kappa \Rightarrow\left\|u_{0}\right\|_{L^{2}\left(\mathbb{T}^{2}\right)}^{2} \leq K \int_{0}^{T}\left\|e^{-i t(-\Delta+V)} u_{0}\right\|_{L^{2}(\Omega)}^{2} d t,
$$

for any $u_{0} \in L^{2}\left(\mathbb{T}^{2}\right)$.

Proof. The Duhamel formula gives

$$
u=e^{-i t(-\Delta+V)} u_{0}=e^{i t \Delta} u_{0}+\frac{1}{i} \int_{0}^{t} e^{i(t-s) \Delta}(V u(s)) d s,
$$

and Jaffard's result (estimate (1.4) for $V=0$ ) applies to the first term. Hence, for a constant $K_{0}$ depending on $\Omega$ and $T$,

$$
\begin{aligned}
\left\|u_{0}\right\|_{L^{2}\left(\mathbb{T}^{2}\right)} \leq & K_{0} \int_{0}^{T}\left\|e^{i t \Delta} u_{0}\right\|_{L^{2}(\Omega)}^{2} d t \\
= & K_{0} \int_{0}^{T}\left\|e^{i t(\Delta-V)} u_{0}-\frac{1}{i} \int_{0}^{t} e^{i(t-s) \Delta}(V u(s)) d s\right\|_{L^{2}(\Omega)}^{2} d t \\
\leq & 2 K_{0} \int_{0}^{T}\left\|e^{i t(\Delta-V)} u_{0}\right\|_{L^{2}(\Omega)}^{2} d t \\
& +2 K_{0} T\left\|\int_{0}^{t} e^{i(t-s) \Delta}(V u(s)) d s\right\|_{L^{\infty}\left((0, T) ; L^{2}\left(\mathbb{T}^{2}\right)\right)}^{2} .
\end{aligned}
$$

We now use Proposition 2.2 with $\mathcal{V}=\{V\}, v_{0}=0$ and $f=V u$ to obtain

$$
\begin{aligned}
\left\|\int_{0}^{t} e^{i(t-s) \Delta}(V u(s)) d s\right\|_{L^{\infty}\left((0, T) ; L^{2}\left(\mathbb{T}^{2}\right)\right)} & \leq C\|V u\|_{L^{4 / 3}\left(\mathbb{T}^{2} ; L^{2}(0, T)\right)} \\
& \leq C\|V\|_{L^{2}\left(\mathbb{T}^{2}\right)}\|u\|_{L^{4}\left(\mathbb{T}^{2} ; L^{2}(0, T)\right)}
\end{aligned}
$$


Applying Proposition 2.2 to the right-hand side, now with $v_{0}=u_{0}, f=0$, gives

$$
\left\|\int_{0}^{t} e^{i(t-s) \Delta}(V u(s)) d s\right\|_{L^{\infty}\left((0, T) ; L^{2}\left(\mathbb{T}^{2}\right)\right)} \leq C\|V\|_{L^{2}\left(\mathbb{T}^{2}\right)}\left\|u_{0}\right\|_{L^{2}\left(\mathbb{T}^{2}\right)},
$$

so that (2.11) becomes

$$
\|u\|_{L^{2}\left(\mathbb{T}^{2}\right)}^{2} \leq 2 K_{0} \int_{0}^{T}\left\|e^{i t(\Delta-V)} u_{0}\right\|_{L^{2}(\Omega)}^{2} d t+2 C K_{0} T\|V\|_{L^{2}\left(\mathbb{T}^{2}\right)}^{2}\left\|u_{0}\right\|_{\left.L^{2}\left(\mathbb{T}^{2}\right)\right)}^{2} .
$$

To conclude, it suffices to take $2 C K_{0} T \kappa^{2} \leq 1 / 2$. (We note that since $K_{0}$ depends on $\Omega$ and $T$ while $C$ depends on $T$, we have no other choice than taking $\kappa>0$ small.)

Remark. In $\S 5$ we will eliminate the smallness assumption on $\|V\|_{L^{2}}$ and that will prove Theorem 2.

The proof of Proposition 2.2 proceeds in several steps. We start by proving the estimate for $V=0$, then we prove the general case by a perturbation argument.

The next proposition is a "fuzzy" version of the classical estimate of Zygmund:

$$
\exists C>0 \forall \tau \in \mathbb{N}, \quad\left\|\sum_{n \in \mathbb{Z}^{2},|n|^{2}=\tau} c_{n} e^{i n \cdot x}\right\|_{L^{4}\left(\mathbb{T}^{2}\right)}^{2} \leq C \sum_{n \in \mathbb{Z}^{2},|n|^{2}=\tau}\left|c_{n}\right|^{2},
$$

and it is motivated by the Córdoba square function estimate [9]:

Proposition 2.4. There exists $C>0$ such that for any $\kappa \geq 0$ and $0<h<1$, and any $u \in L^{2}\left(\mathbb{T}^{2}\right)$ satisfying

$$
\hat{u}(n)=0 \quad \text { for } n \notin \mathcal{B}(\kappa, h):=\left\{n \in \mathbb{Z}^{2} ;\left.\left|h^{2}\right| n\right|^{2}-1 \mid \leq \kappa^{2} h^{2}\right\},
$$

we have

$$
\|u\|_{L^{4}\left(\mathbb{T}^{2}\right)} \leq \begin{cases}C(1+\kappa)^{1 / 4}\left(1+\kappa^{2} h\right)^{1 / 4}\|u\|_{L^{2}\left(\mathbb{T}^{2}\right)} & \text { if } \kappa \leq h^{-1} \\ C(1+\kappa)^{1 / 2}\|u\|_{L^{2}\left(\mathbb{T}^{2}\right)} & \text { if } \kappa \geq h^{-1}\end{cases}
$$

We note that the case of $\kappa=0$ in (2.13) is (2.12), while $\kappa=h^{-1}$ is simply Sobolev embeddings and $\kappa=h^{-1 / 2}$ is Sogge's estimate for spectral projectors [22], [23, Theorem 10.11] (for which we give an arithmetic proof below).

Proof. We first note that we can assume that $\kappa \geq 1$ as the sets $\mathcal{B}(\kappa, h)$ increase with increasing $\kappa$.

For a constant $\delta>0$, to be fixed later, we distinguish two regimes: $\kappa h \geq \delta$ and $\kappa h \leq \delta$. In the first regime, the estimate follows from the Sobolev embedding $H^{1 / 2}\left(\mathbb{T}^{2}\right) \rightarrow L^{4}\left(\mathbb{T}^{2}\right): \hat{u}(n)=0$ unless $|n|^{2} \leq h^{-2}+\kappa^{2} \leq(1 / \delta+1) \kappa^{2}$, and this implies

$$
\|u\|_{H^{1 / 2}\left(\mathbb{T}^{2}\right)} \leq C_{\delta} \kappa^{1 / 2}\|u\|_{L^{2}} .
$$

From now on we assume that $h \kappa \leq \delta$. In this regime, we can change the set $\mathcal{B}(\kappa, h)$ to

$$
\mathcal{A}(\kappa, h):=\left\{n \in \mathbb{Z}^{2} ;|h| n|-1| \leq \kappa^{2} h^{2}\right\}
$$


The idea is to prove an arithmetic version of the Córdoba square function estimate [9]. Indeed, the usual version allows one only to work with $\kappa \geq h^{-1 / 2}$ (the uncertainty principle). Our version below allows us to get estimates all the way down to $\kappa \sim 1$ (that is, much beyond the uncertainty principle). We first notice that we can also assume that the spectrum of $u$ is also contained in the upper quadrant $\{z \in \mathbb{C} ; \operatorname{Re} z \geq 0, \operatorname{Im} z \geq 0\}$ of the plane (here and in what follows we identify $\mathbb{R}^{2}$ with $\mathbb{C}$ ). Indeed, if the result is true for the upper quadrant, by symmetry, it is true for any quadrant, and with a different constant in the general case. Then we decompose the intersection of the annulus with this quadrant into a disjoint union of angular sectors of angles $h \kappa$ :

$$
\mathcal{A}(\kappa, h) \cap\{\operatorname{Im} z \geq 0, \operatorname{Re} z \geq 0\}=\bigcup_{\alpha=0}^{N_{\kappa, h}} \mathcal{A}_{\alpha}(\kappa, h), \quad N_{\kappa, h}:=\left[\frac{\pi}{2 h \kappa}\right],
$$

where

$$
\mathcal{A}_{\alpha}(\kappa, h):=\left\{z ; \operatorname{Re} z \geq 0, \operatorname{Im} z \geq 0,|h| z|-1| \leq \kappa^{2} h^{2}, \arg (z) \in[\alpha h \kappa,(\alpha+1) h \kappa)\right\} .
$$

The proof relies on the following geometric lemma which will be proved in Appendix B:

Lemma 2.5. Fix $\delta>0$ small enough. Then there exists $Q \in \mathbb{N}$ such that for any $0<$ $h<1$ and any $1 \leq \kappa \leq \delta / h$, we have

$$
\begin{aligned}
& \forall \alpha, \beta, \alpha^{\prime}, \beta^{\prime} \in\left\{0,1, \ldots, N_{\kappa, h}\right\}, \\
&\left(\mathcal{A}_{\alpha}(\kappa, h)+\mathcal{A}_{\beta}(\kappa, h)\right) \cap\left(\mathcal{A}_{\alpha^{\prime}}(\kappa, h)+\mathcal{A}_{\beta^{\prime}}(\kappa, h)\right) \neq \emptyset \\
& \quad \Rightarrow\left|\alpha-\alpha^{\prime}\right|+\left|\beta-\beta^{\prime}\right| \leq Q \text { or }\left|\alpha-\beta^{\prime}\right|+\left|\beta-\alpha^{\prime}\right| \leq Q .
\end{aligned}
$$

We apply the lemma as folllows. We have

$$
u=\sum_{\alpha=0}^{N_{\kappa, h}} U_{\alpha}, \quad u^{2}=\sum_{\alpha, \beta=0}^{N_{\kappa, h}} U_{\alpha} U_{\beta}, \quad U_{\alpha}:=\sum_{n \in \mathbb{Z}^{2} \cap \mathcal{A}_{\alpha}(\kappa, h)} u_{n} e^{i n \cdot x},
$$

and hence

$$
\|u\|_{L^{4}\left(\mathbb{T}^{2}\right)}^{4}=\sum_{\alpha, \beta, \alpha^{\prime}, \beta^{\prime}=0}^{N_{\kappa, h}} \int_{\mathbb{T}^{2}} U_{\alpha} U_{\beta} \bar{U}_{\alpha^{\prime}} \bar{U}_{\beta^{\prime}}(x) d x .
$$

The integral vanishes unless

$$
\left(\mathcal{A}_{\alpha}(\kappa, h)+\mathcal{A}_{\beta}(\kappa, h)\right) \cap\left(\mathcal{A}_{\alpha^{\prime}}(\kappa, h)+\mathcal{A}_{\beta^{\prime}}(\kappa, h)\right) \neq \emptyset
$$

as otherwise

$n \in \mathbb{Z}^{2} \cap \mathcal{A}_{\alpha}, m \in \mathbb{Z}^{2} \cap \mathcal{A}_{\beta}, p \in \mathbb{Z}^{2} \cap \mathcal{A}_{\alpha^{\prime}}, q \in \mathbb{Z}^{2} \cap \mathcal{A}_{\beta^{\prime}} \Rightarrow n+m-(p+q) \neq 0$,

and, using the inner product (2.7), $\int_{\mathbb{T}^{2}} e^{i x \cdot(n+m-p-q)} d x=0$. Lemma 2.5 then shows that we can restrict the sum in (2.15) to the subset of indices $\left(\alpha, \beta, \alpha^{\prime}, \beta^{\prime}\right)$ satisfying

$$
\left|\alpha-\alpha^{\prime}\right|+\left|\beta-\beta^{\prime}\right| \leq Q \quad \text { or } \quad\left|\alpha-\beta^{\prime}\right|+\left|\beta-\alpha^{\prime}\right| \leq Q .
$$


This and an application of Hölder's inequality,

$$
\begin{aligned}
\left|\int_{\mathbb{T}^{2}} U_{\alpha} U_{\beta} \bar{U}_{\alpha^{\prime}} \bar{U}_{\beta^{\prime}}(x) d x\right| & \leq\left\|U_{\alpha}\right\|_{L^{4}\left(\mathbb{T}^{2}\right)}\left\|U_{\beta}\right\|_{L^{4}\left(\mathbb{T}^{2}\right)}\left\|U_{\alpha^{\prime}}\right\|_{L^{4}\left(\mathbb{T}^{2}\right)}\left\|U_{\beta^{\prime}}\right\|_{L^{4}\left(\mathbb{T}^{2}\right)} \\
& \leq\left\{\begin{array}{l}
\left(\left\|U_{\alpha}\right\|_{L^{4}\left(\mathbb{T}^{2}\right)}^{2}+\left\|U_{\alpha^{\prime}}\right\|_{L^{4}\left(\mathbb{T}^{2}\right)}^{2}\right)\left(\left\|U_{\beta}\right\|_{L^{4}\left(\mathbb{T}^{2}\right)}^{2}+\left\|U_{\beta^{\prime}}\right\|_{L^{4}\left(\mathbb{T}^{2}\right)}^{2}\right) \\
\left(\left\|U_{\alpha}\right\|_{L^{4}\left(\mathbb{T}^{2}\right)}^{2}+\left\|U_{\beta^{\prime}}\right\|_{L^{4}\left(\mathbb{T}^{2}\right)}^{2}\right)\left(\left\|U_{\beta}\right\|_{L^{4}\left(\mathbb{T}^{2}\right)}^{2}+\left\|U_{\alpha^{\prime}}\right\|_{L^{4}\left(\mathbb{T}^{2}\right)}^{2}\right)
\end{array}\right.
\end{aligned}
$$

give

$$
\|u\|_{L^{4}\left(\mathbb{T}^{2}\right)}^{4} \leq C Q^{2}\left(\sum_{\alpha=0}^{N_{\kappa, h}}\left\|U_{\alpha}\right\|_{L^{4}\left(\mathbb{T}^{2}\right)}^{2}\right)^{2} .
$$

To estimate the norms of $U_{\alpha}$ we write

$$
\begin{aligned}
\left\|U_{\alpha}\right\|_{L^{4}\left(\mathbb{T}^{2}\right)} & \leq C\left\|U_{\alpha}\right\|_{L^{\infty}\left(\mathbb{T}^{2}\right)}^{1 / 2}\left\|U_{\alpha}\right\|_{L^{2}\left(\mathbb{T}^{2}\right)}^{1 / 2} \\
& \leq\left(\sum_{n \in \mathbb{Z}^{2} \cap \mathcal{A}_{\alpha}(\kappa, h)}\left|u_{n}\right|\right)^{1 / 2}\left(\sum_{n \in \mathbb{Z}^{2} \cap \mathcal{A}_{\alpha}(\kappa, h)}\left|u_{n}\right|^{2}\right)^{1 / 4} \\
& \leq C\left|\mathbb{Z}^{2} \cap \mathcal{A}_{\alpha}(\kappa, h)\right|^{1 / 4}\left\|U_{\alpha}\right\|_{L^{2}\left(\mathbb{T}^{2}\right)}
\end{aligned}
$$

To estimate the number of integral points in $\mathcal{A}_{\alpha}(\kappa, h)$, we first notice that $\mathcal{A}_{\alpha}(\kappa, h)$ is included in a rectangle of height $1+\kappa$ and width $1+3 \kappa^{2} h$.

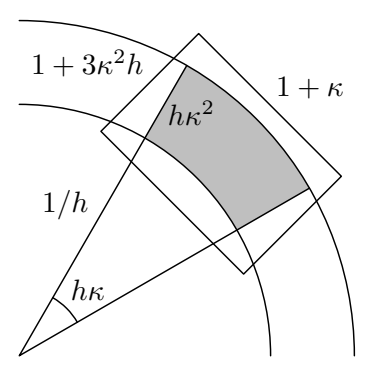

Fig. 1. The angular region $\mathcal{A}_{\alpha}(\kappa, h)$ fitted inside a rectangle.

Now, the number of integral points in any rectangle of height $H$ and width $W$ is bounded by $C \max (H, 1) \max (W, 1)$. (To see this, notice that open discs of radius $1 / 2$ centered at the integer points are pairwise disjoint and are all included in a rectangle of height $H+1$ and width $W+1$.) Hence, recalling that $\kappa h \leq \delta$, we have

$$
\left|\mathbb{Z}^{2} \cap \mathcal{A}_{\alpha}(\kappa, h)\right| \leq C(1+\kappa)\left(1+3 \kappa^{2} h\right) \leq C(1+\kappa)^{2} .
$$

Combining this with (2.17) and (2.16) gives

$$
\|u\|_{L^{4}\left(\mathbb{T}^{2}\right)}^{4} \leq C(1+\kappa)\left(1+\kappa^{2} h\right)\|u\|_{L^{2}\left(\mathbb{T}^{2}\right)}^{4},
$$

concluding the proof. 
The next step in the proof of Proposition 2.2 is an optimal (at least in terms of the spectral region where it holds) resolvent estimate-see Kenig-Dos Santos-Salo [11, Remark 1.2] and Bourgain-Shao-Sogge-Yao [3] for related results.

Proposition 2.6. For any compact subset $\mathcal{V} \subset L^{2}\left(\mathbb{T}^{2}\right)$, there exist $C(\mathcal{V})$ and $\epsilon>0$ such that for any $V \in \mathcal{V}+B(0, \epsilon)$, any $f \in C^{\infty}\left(\mathbb{T}^{2}\right)$ and any $\tau \in \mathbb{C}$ with $|\operatorname{Im} \tau| \geq 1$,

$$
\left\|(-\Delta+V-\tau)^{-1} f\right\|_{L^{4}\left(\mathbb{T}^{2}\right)} \leq C\|f\|_{L^{4 / 3}\left(\mathbb{T}^{2}\right)} .
$$

We deduce it from Proposition 2.4 and the following elementary result:

Lemma 2.7. Assume that $\mathcal{V}$ is a compact subset of $L^{2}\left(\mathbb{T}^{2}\right)$. Then for any $\delta>0$ there exists $C_{\delta}>0$ and for any $V \in \mathcal{V}$ there exists $V_{\delta} \in L^{\infty}\left(\mathbb{T}^{2}\right)$ such that

$$
\left\|V_{\delta}-V\right\|_{L^{2}\left(\mathbb{T}^{2}\right)} \leq \delta, \quad\left\|V_{\delta}\right\|_{L^{\infty}\left(\mathbb{T}^{2}\right)} \leq C_{\delta} .
$$

Proof. This is obvious for $\mathcal{V}=\left\{V_{0}\right\}$ since $L^{\infty} \subset L^{2}$ is dense. Applying it with $\delta$ replaced by $\delta / 2$ the statement remains true for $V$ with $\left\|V-V_{0}\right\|_{L^{2}} \leq \delta / 2$. A covering argument provides the result for a general compact set in $L^{2}$.

Proof of Proposition 2.6. For $\operatorname{Re} \tau \leq C$ for any fixed $C$, we get (2.18) directly. Indeed, from $(-\Delta-\tau+V) u=f$, multiplying by $\bar{u}$, integrating by parts and taking real and imaginary parts, we get

$$
\begin{aligned}
\|\nabla u\|_{L^{2}\left(\mathbb{T}^{2}\right)}^{2}-\operatorname{Re} \tau\|u\|_{L^{2}\left(\mathbb{T}^{2}\right)}^{2} & \leq\left\|V|u|^{2}\right\|_{L^{1}\left(\mathbb{T}^{2}\right)}+\|u\|_{L^{4}\left(\mathbb{T}^{2}\right)}\|f\|_{L^{4 / 3}\left(\mathbb{T}^{2}\right)}, \\
|\operatorname{Im} \tau|\|u\|_{L^{2}\left(\mathbb{T}^{2}\right)}^{2} & \leq\|u\|_{L^{4}\left(\mathbb{T}^{2}\right)}\|f\|_{L^{4 / 3}\left(\mathbb{T}^{2}\right)} .
\end{aligned}
$$

Since $|\operatorname{Im} \tau| \geq 1$, the Sobolev embedding and Lemma 2.7 imply

$$
\begin{aligned}
\|u\|_{L^{4}\left(\mathbb{T}^{2}\right)}^{2} & \leq C\|u\|_{H^{1}\left(\mathbb{T}^{2}\right)}^{2} \\
\leq & C\left(\left\|V_{\delta}-V\right\|_{L^{2}\left(\mathbb{T}^{2}\right)}\|u\|_{L^{4}\left(\mathbb{T}^{2}\right)}^{2}+\left\|V_{\delta}\right\|_{L^{\infty}\left(\mathbb{T}^{2}\right)}\|u\|_{L^{2}\left(\mathbb{T}^{2}\right)}^{2}+\|u\|_{L^{4}\left(\mathbb{T}^{2}\right)}\|f\|_{L^{4 / 3}\left(\mathbb{T}^{2}\right)}\right) \\
\leq & C(\delta+\epsilon)\|u\|_{L^{4}\left(\mathbb{T}^{2}\right)}^{2}+C\left(\left\|V_{\delta}\right\|_{L^{\infty}\left(\mathbb{T}^{2}\right)}+1\right)\|u\|_{L^{4}\left(\mathbb{T}^{2}\right)}\|f\|_{L^{4 / 3}\left(\mathbb{T}^{2}\right)}
\end{aligned}
$$

and choosing $\epsilon<\delta=\frac{1}{4} C$ gives the result.

For $\operatorname{Re} \tau>C$ we start with the case of $V=0$ and notice that

$$
(-\Delta-\tau)^{-1}=(-\Delta-\tau)^{-1 / 2}\left((-\Delta-\bar{\tau})^{-1 / 2}\right)^{*}: L^{4 / 3} \rightarrow L^{4}
$$

follows from $(-\Delta-\tau)^{-1 / 2}: L^{2} \rightarrow L^{4}=\left(L^{4 / 3}\right)^{*}$. Here the square root is defined using the spectral theorem and the branches chosen for $\pm \operatorname{Im} \tau>1$ so that

$$
(\lambda-\tau)^{1 / 2} \overline{(\lambda-\bar{\tau})^{1 / 2}}=\lambda-\tau, \quad \lambda \geq 0
$$

Hence we need to prove that

$$
\|u\|_{L^{4}\left(\mathbb{T}^{2}\right)} \leq C\|f\|_{L^{2}\left(\mathbb{T}^{2}\right)}, \quad u:=(-\Delta-\tau)^{-1 / 2} f .
$$


To use Proposition 2.4 we write the resolvent applied to $f$ using the Fourier series

$u=\sum_{n} \frac{f_{n}}{\left(|n|^{2}-\tau\right)^{1 / 2}} e^{i n \cdot x}=u_{0}+\sum_{j=1}^{\infty} u_{j}, \quad u_{j}:=\sum_{2^{j-1} \leq\left.|| n\right|^{2}-\operatorname{Re} \tau \mid<2^{j}} \frac{f_{n}}{\left(|n|^{2}-\tau\right)^{1 / 2}} e^{i n \cdot x}$.

We note that $u_{0}=\sum_{\left.|| n\right|^{2}-\operatorname{Re} \tau \mid<1} f_{n}\left(|n|^{2}-\tau\right)^{-1 / 2} e^{i n \cdot x}$ and hence Proposition 2.4 gives

$$
\left\|u_{0}\right\|_{L^{4}\left(\mathbb{T}^{2}\right)} \leq C\|f\|_{L^{2}\left(\mathbb{T}^{2}\right)} .
$$

Applying (2.13) to $u_{j}$ 's with $h=(\operatorname{Re} \tau)^{-1 / 2}$ and $\kappa=2^{j / 2}$ gives

$$
\begin{aligned}
\left\|u-u_{0}\right\|_{L^{4}\left(\mathbb{T}^{2}\right)} & \leq C \sum_{j} 2^{j / 4}\left\|u_{j}\right\|_{L^{2}\left(\mathbb{T}^{2}\right)} \\
& \leq\left(\sum_{j=1}^{\infty} 2^{-j / 2}\right)^{1 / 2}\left(\sum_{j=1}^{\infty} 2^{j} \sum_{2^{j-1} \leq|n|^{2}-\operatorname{Re} \tau \mid<2^{j}} \frac{\left|f_{n}\right|^{2}}{\left.|| n\right|^{2}-\tau \mid}\right)^{1 / 2} \\
& \leq C\|f\|_{L^{2}\left(\mathbb{T}^{2}\right)}
\end{aligned}
$$

which concludes the proof of Proposition 2.6 for $V=0$.

The general case $V \neq 0$ follows from the same perturbation argument as in the case $\operatorname{Re} \tau \leq C$. Indeed, from $(-\Delta-\tau) u=-V u+f$, we deduce

$$
|\operatorname{Im} \tau|\|u\|_{L^{2}\left(\mathbb{T}^{2}\right)}^{2} \leq\|u\|_{L^{4}\left(\mathbb{T}^{2}\right)}\|f\|_{L^{4 / 3}\left(\mathbb{T}^{2}\right)},
$$

and from the resolvent estimate for $V=0$,

$$
\begin{aligned}
\|u\|_{L^{4}\left(\mathbb{T}^{2}\right)} & \leq C\|V u\|_{L^{4 / 3}\left(\mathbb{T}^{2}\right)}+\|f\|_{L^{4 / 3}\left(\mathbb{T}^{2}\right)} \\
& \leq C\left(\left\|V_{\delta}-V\right\|_{L^{2}\left(\mathbb{T}^{2}\right)}\|u\|_{L^{4}\left(\mathbb{T}^{2}\right)}+\left\|V_{\delta}\right\|_{L^{\infty}\left(\mathbb{T}^{2}\right)}\|u\|_{L^{2}\left(\mathbb{T}^{2}\right)}+\|f\|_{L^{4 / 3}\left(\mathbb{T}^{2}\right)}\right) \\
& \leq C \delta\|u\|_{L^{4}\left(\mathbb{T}^{2}\right)}+C\left(\left\|V_{\delta}\right\|_{L^{\infty}\left(\mathbb{T}^{2}\right)}\|u\|_{L^{4}\left(\mathbb{T}^{2}\right)}^{1 / 2}\|f\|_{L^{4 / 3}\left(\mathbb{T}^{2}\right)}^{1 / 2}+\|f\|_{L^{4 / 3}\left(\mathbb{T}^{2}\right)}\right) .
\end{aligned}
$$

Choosing $\delta$ small enough gives the desired estimate.

Proof of Proposition 2.2. Let us first study the contribution of $v_{0}$. Putting $T u_{0}=$ $e^{i t(\Delta-V)} u_{0}$ we have

$T T^{*} f=\int_{0}^{T} e^{i(t-s)(\Delta-V)} f(s) d s=\int_{0}^{t} e^{i(t-s)(\Delta-V)} f(s) d s+\int_{t}^{T} e^{i(t-s)(\Delta-V)} f(s) d s$.

To prove that $T: L^{2}\left(\mathbb{T}^{2}\right) \rightarrow L^{4}\left(\mathbb{T}_{x}^{2} ; L^{2}(0, T)\right)$ it suffices to prove that

$$
T T^{*}: L^{4 / 3}\left(\mathbb{T}_{x}^{2} ; L^{2}(0, T)\right) \rightarrow L^{4}\left(\mathbb{T}_{x}^{2} ; L^{2}(0, T)\right),
$$

and we will show it for the two operators on the right-hand side, say the first one. That means showing that for solutions to $\left(i \partial_{t}+\Delta-V\right) v=f,\left.v\right|_{t=0}=0$, we have

$$
\|v\|_{L^{4}\left(\mathbb{T}^{2} ; L^{2}(0, T)\right)} \leq C\|f\|_{L^{4 / 3}\left(\mathbb{T}^{2} ; L^{2}(0, T)\right)} .
$$


Let $U=v e^{-t} \mathbb{1}_{t>0}$ and $F=f e^{-t} \mathbb{1}_{0<t<T}$. We have $\left(i \partial_{t}+\Delta-V+i\right) U=F$ and hence, by taking the Fourier transform in $t$,

$$
(\Delta-V+i-\tau) \widehat{U}=\widehat{F} .
$$

Proposition 2.6 now shows that for any $\tau \in \mathbb{R}$,

$$
\|\widehat{U}(\tau)\|_{L^{4}\left(\mathbb{T}^{2}\right)} \leq C\|\widehat{F}(\tau)\|_{L^{4 / 3}\left(\mathbb{T}^{2}\right)},
$$

which implies

$$
\begin{aligned}
\|u\|_{L^{4}\left(\mathbb{T}_{x}^{2} ; L^{2}(0, T)\right)} & \leq C\|U\|_{L^{4}\left(\mathbb{T}_{x}^{2} ; L^{2}\left(\mathbb{R}_{t}\right)\right)}=C\|\widehat{U}\|_{L^{4}\left(\mathbb{T}_{x}^{2} ; L^{2}\left(\mathbb{R}_{\tau}\right)\right)} \\
& \leq C\|\widehat{U}\|_{L^{2}\left(\mathbb{R}_{\tau} ; L^{4}\left(\mathbb{T}_{x}^{2}\right)\right)} \leq C^{\prime}\|\widehat{F}\|_{L^{2}\left(\mathbb{R}_{\tau} ; L^{4 / 3}\left(\mathbb{T}_{x}^{2}\right)\right)} \\
& \leq C^{\prime}\|\widehat{F}\|_{L^{4 / 3}\left(\mathbb{T}_{x}^{2} ; L^{2}\left(\mathbb{R}_{\tau}\right)\right)}=C^{\prime}\|F\|_{L^{4 / 3}\left(\mathbb{T}_{x}^{2} ; L^{2}(0, T)\right)}
\end{aligned}
$$

concluding the proof of (2.19).

Part of the nonhomogeneous estimate in (2.10),

$$
\|v\|_{L^{\infty}\left((0, T) ; L^{2}\left(\mathbb{T}^{2}\right)\right) \cap L^{4}\left(\mathbb{T}_{x}^{2} ; L^{2}(0, T)\right)} \leq C\|f\|_{L^{1}\left((0, T) ; L^{2}\left(\mathbb{T}^{2}\right)\right)},
$$

follows from the boundedness of the operator $T$ from $L^{2}$ to $L^{4}\left(\mathbb{T}^{2} ; L^{2}(0, T)\right)$ and the Minkowski inequality. Finally, since the dual of the operator $f \mapsto \int_{0}^{t} e^{i(t-s)(\Delta-V)} f(s) d s$ is $g \mapsto \int_{t}^{T} e^{i(t-s)(\Delta-V)} g(s) d s$, we also get

$$
\|u\|_{L^{\infty}\left((0, T) ; L^{2}\left(\mathbb{T}^{2}\right)\right)} \leq C\|f\|_{L^{1}\left((0, T) ; L^{2}\left(\mathbb{T}^{2}\right)\right)+L^{4 / 3}\left(\mathbb{T}^{2} ; L^{2}(0, T)\right)},
$$

which concludes the proof of Proposition 2.2.

We conclude this section with a continuity result which will be useful later:

Proposition 2.8. Consider a sequence $\left\{V_{n}\right\}_{n \in \mathbb{N}} \subset L^{2}\left(\mathbb{T}^{2}\right)$ converging to $V \in L^{2}\left(\mathbb{T}^{2}\right)$. Then there exists $C>0$ such that for any $v_{0} \in L^{2}\left(\mathbb{T}^{2}\right)$,

$$
\left\|e^{-i t(-\Delta+V)} v_{0}-e^{-i t\left(-\Delta+V_{n}\right)} v_{0}\right\|_{L^{\infty}\left((0, T) ; L^{2}\left(\mathbb{T}^{2}\right)\right)} \leq C\left\|V-V_{n}\right\|_{L^{2}\left(\mathbb{T}^{2}\right)}\left\|u_{0}\right\|_{L^{2}\left(\mathbb{T}^{2}\right)} .
$$

Remark. The result in Proposition 2.8 can be stated more generally, for a compact subset $\mathcal{V} \subset L^{2}\left(\mathbb{T}^{2}\right)$, and is equivalent to the Lipschitz continuity of the map

$$
V \in \mathcal{V} \subset L^{2}\left(\mathbb{T}^{2}\right) \mapsto e^{-i t(-\Delta+V)} \in L^{\infty}\left((0, T) ; \mathcal{L}\left(L^{2}\left(\mathbb{T}^{2}\right)\right)\right) .
$$

A slight modification of the proof presented here shows that it is in fact also Lipschitz on bounded subsets of $L^{p}, p>2$. It would be interesting to investigate such properties on other manifolds, as they seem to depend strongly on the geometry. Indeed, the analysis in [5, Theorem 2] is likely to give that on spheres, there exists a sequence of potentials $\left\{V_{n}\right\}_{n \in \mathbb{N}}$ such that for any $T>0$ and $p<\infty$,

$$
\lim _{n \rightarrow \infty}\left\|V_{n}\right\|_{L^{p}\left(\mathbb{S}^{2}\right)}=0, \quad \text { but } \quad \lim _{n \rightarrow \infty}\left\|e^{i t \Delta}-e^{i t\left(\Delta-V_{n}\right)}\right\|_{L^{\infty}\left((0, T) ; \mathcal{L}\left(L^{2}\left(\mathbb{S}^{2}\right)\right)\right)}>0 .
$$


Proof of Proposition 2.8. Let $u=e^{i t(\Delta-V)} v_{0}$ and $u_{n}=e^{i t\left(\Delta-V_{n}\right)} v_{0}$, so that the Duhamel formula gives

$$
u-u_{n}=\frac{1}{i} \int_{0}^{t} e^{i(t-s)(\Delta-V)}\left(\left(V_{n}-V\right) u_{n}(s)\right) d s .
$$

Proposition 2.2 applied with $\mathcal{V}=\{V\}, v_{0}=0$ and $f=\left(V_{n}-V\right) u_{n}$, and Hölder's inequality give

$$
\begin{aligned}
\left\|u_{V}-u_{n}\right\|_{L^{\infty}\left((0, T) ; L^{2}\left(\mathbb{T}_{x}^{2}\right)\right)} & \leq C\left\|\left(V-V_{n}\right) u_{n}\right\|_{L^{4 / 3}\left(\mathbb{T}^{2} ; L^{2}(0, T)\right)} \\
& \leq C\left\|V-V_{n}\right\|_{L^{2}\left(\mathbb{T}^{2}\right)}\left\|u_{n}\right\|_{L^{4}\left(\mathbb{T}^{2} ; L^{2}(0, T)\right)} .
\end{aligned}
$$

Applying Proposition 2.2 again, now with $\mathcal{V}=\left\{V_{n} ; n \in \mathbb{N}\right\} \cup\{V\}$, and $f=0$, we estimate the right-hand side to obtain the desired estimate:

$$
\left\|u_{V}-u_{n}\right\|_{L^{\infty}\left((0, T) ; L^{2}\left(\mathbb{T}_{x}^{2}\right)\right)} \leq C\left\|V-V_{n}\right\|_{L^{2}\left(\mathbb{T}^{2}\right)}\left\|v_{0}\right\|_{L^{2}\left(\mathbb{T}_{x}^{2}\right)} .
$$

\section{One-dimensional observability estimates}

In this section we consider the one-dimensional analog of our result which we prove for $L^{p}$ potentials, $p>1$. In applications to control and observability on 2-tori we will use it only for $p=2$ but the finer estimate may be of independent interest.

Let us recall (see Section 2.1) that the operator $-\partial_{x}^{2}+W, W \in L^{1}\left(\mathbb{T}^{1}\right)$, which is defined on $C^{\infty}\left(\mathbb{T}^{1}\right)$ has a unique self-adjoint extension with the domain containing $H^{1}\left(\mathbb{T}^{1}\right)$ (if $W \in L^{2}\left(\mathbb{T}^{1}\right)$ the domain is $H^{2}\left(\mathbb{T}^{1}\right)$ ). The resolvent $\left(-\partial_{x}^{2}+W-z\right)^{-1}, z \notin \mathbb{R}$, is compact and the spectrum is discrete with eigenvalues $\lambda_{j} \rightarrow \infty$.

We have the following one-dimensional observability result which holds for functions satisfying the Floquet boundary conditions:

Proposition 3.1. Assume that $W \in L^{p}\left(\mathbb{T}^{1}\right), p>1$, and $\omega \subset \mathbb{T}^{1}$ is a non-empty open set. Then for any $T>0$ there exists $K_{0}>0$ such that for any $k \in \mathbb{R}$ and $v \in L^{2}\left(\mathbb{T}^{1}\right)$,

$$
\|v\|_{L^{2}\left(\mathbb{T}^{1}\right)}^{2} \leq K_{0} \int_{0}^{T}\left\|e^{i t\left(\left(\partial_{x}+i k\right)^{2}-W\right)} v\right\|_{L^{2}(\omega)}^{2} d t .
$$

Let us first notice that conjugating with $e^{i x[k]}$, we can replace $k$ by $k-[k]$ and hence assume that $k \in[0,1]$. We first prove the stationary version following the elementary approach of [7]:

Proposition 3.2. Under the assumptions of Proposition 3.1 there exists $C_{1}=$ $C_{1}\left(\omega,\|W\|_{L^{p}\left(\mathbb{T}^{1}\right)}\right)$ such that for any $\tau \in \mathbb{R}$, any solution to

$$
\left(-\left(\partial_{x}+i k\right)^{2}+W-\tau\right) u=g
$$

satisfies

$$
\|u\|_{L^{2}\left(\mathbb{T}^{1}\right)} \leq C_{1}\left(\langle\tau\rangle^{-1 / 2}\|g\|_{L^{2}\left(\mathbb{T}^{1}\right)}+\|u\|_{L^{2}(\omega)}\right) .
$$

This follows from the following result which holds for $W=0$. 
Lemma 3.3. Let $\omega \subset \mathbb{T}^{1}$ be an open set. Then there exists a constant $C_{0}=C_{0}(\omega)$ such that for any $k \in \mathbb{R}$ and any $u \in H^{1}\left(\mathbb{T}^{1}\right)$ satisfying

$$
\left(-\left(\partial_{x}+i k\right)^{2}-\tau\right) u=f+g,
$$

we have

$$
\begin{aligned}
\|u\|_{L^{2}\left(\mathbb{T}^{1}\right)}+\langle\tau\rangle^{-1 / 2}\left\|\partial_{x} u\right\|_{L^{2}\left(\mathbb{T}^{1}\right)} & \\
& \leq C_{0}\left(\|f\|_{H^{-1}\left(\mathbb{T}^{1}\right)}+\langle\tau\rangle^{-1 / 2}\|g\|_{L^{2}\left(\mathbb{T}^{1}\right)}+\|u\|_{L^{2}(\omega)}\right) .
\end{aligned}
$$

Proof. We start by showing that there exists a constant $C$ such that for any $k \in \mathbb{R}$ and any $u \in H^{1}\left(\mathbb{T}^{1}\right)$ satisfying

$$
\left(-\left(\partial_{x}+i k\right)^{2}-\tau\right) u=\left(\partial_{x}+i k\right) f+g
$$

we have

$$
\|u\|_{L^{2}\left(\mathbb{T}^{1}\right)} \leq C\left(\|f\|_{L^{2}\left(\mathbb{T}^{1}\right)}+\langle\tau\rangle^{-1 / 2}\|g\|_{L^{2}\left(\mathbb{T}^{1}\right)}+\|u\|_{L^{2}(\omega)}\right) .
$$

The elementary proof given in [7] shows that it is true for $k=0$. For any solution $U$ to (3.5), let $v=e^{-i k x} u$, which is no longer periodic but satisfies the Floquet conditions (2.1) and

$$
\left(-\partial_{x}^{2}-\tau\right) v=\partial_{x} F+G, \quad F=e^{-i k x} f, \quad G=e^{-i k x} g .
$$

Choosing a parametrization on $\mathbb{T}^{1}$ so that $2 \pi \in \omega$ we take $\chi \in C^{\infty}\left(\mathbb{T}^{1}\right)$ equal to one in a neighbourhood of $\mathbb{T}^{1} \backslash \omega$, and vanishing in a neighbourhood of $2 \pi$. Hence, supp $\chi v \subset(\epsilon, 2 \pi-\epsilon)$ and $u=\chi v$ defines a function on $\mathbb{T}^{1}$ such that

$$
\left(-\partial_{x}^{2}-\tau\right) u=\partial_{x}\left(\chi F+2 \chi^{\prime} v\right)+\chi G-\chi^{\prime} F-\chi^{\prime \prime} v .
$$

Applying (3.6) for $k=0$, we obtain, using the properties of $\chi$,

$$
\begin{aligned}
\|\chi v\|_{L^{2}\left(\mathbb{T}^{1}\right)} & \leq C\left(\left\|\chi F+2 \chi^{\prime} v\right\|_{L^{2}\left(\mathbb{T}^{1}\right)}+\langle\tau\rangle^{-1 / 2}\left\|\chi G-\chi^{\prime} F-\chi^{\prime \prime} v\right\|_{L^{2}\left(\mathbb{T}^{1}\right)}\right) \\
& \leq C^{\prime}\left(\|F\|_{L^{2}\left(\mathbb{T}^{1}\right)}+\langle\tau\rangle^{-1 / 2}\|G\|_{L^{2}\left(\mathbb{T}^{1}\right)}+\|v\|_{L^{2}(\omega)}\right),
\end{aligned}
$$

which, coming back to $u$, implies that (3.6) holds for any $k$.

Since for $k \in[0,1]$,

$$
\begin{aligned}
\|f\|_{H^{-1}} & =\inf \left\{\|F\|_{L^{2}}+\|i k F+H\|_{L^{2}} ; f=\left(\partial_{x}+i k\right) F+H\right\} \\
& \geq \frac{1}{2} \inf \left\{\|F\|_{L^{2}}+\|H\|_{L^{2}} ; f=\left(\partial_{x}+i k\right) F+H\right\},
\end{aligned}
$$

the estimate on $\|u\|_{L^{2}\left(\mathbb{T}^{1}\right)}, u(x)=e^{i k x} v(x)$, in (3.4) follows from (3.6).

To estimate $\left(\partial_{x}+i k\right) u$ we write

$$
\begin{aligned}
\left\|\left(\partial_{x}+i k\right) u\right\|_{L^{2}\left(\mathbb{T}^{1}\right)}^{2} & =\left\langle\left(-\left(\partial_{x}+i k\right)^{2}-\tau\right) u, u\right\rangle_{L^{2}\left(\mathbb{T}^{1}\right)}+\tau\|u\|_{L^{2}\left(\mathbb{T}^{1}\right)}^{2} \\
& =\langle f+g, u\rangle_{L^{2}\left(\mathbb{T}^{1}\right)}+\tau\|u\|_{L^{2}\left(\mathbb{T}^{1}\right)}^{2} \\
& \leq\|f\|_{H^{-1}\left(\mathbb{T}^{1}\right)}\|u\|_{H^{1}\left(\mathbb{T}^{1}\right)}+\|g\|_{L^{2}\left(\mathbb{T}^{1}\right)}\|u\|_{L^{2}\left(\mathbb{T}^{1}\right)}+\langle\tau\rangle\|u\|_{L^{2}\left(\mathbb{T}^{1}\right)}^{2} \\
& \leq \frac{1}{2}\left\|\left(\partial_{x}+i k\right) u\right\|_{L^{2}\left(\mathbb{T}^{1}\right)}^{2}+C\|f\|_{H^{-1}\left(\mathbb{T}^{1}\right)}^{2}+C\|g\|_{L^{2}\left(\mathbb{T}^{1}\right)}^{2}+C\langle\tau\rangle\|u\|_{L^{2}\left(\mathbb{T}^{1}\right)}^{2} .
\end{aligned}
$$

Using the estimate for $\|u\|_{L^{2}\left(\mathbb{T}^{1}\right)}$ and the fact that $k \in[0,1]$ we obtain (3.4). 
Proof of Proposition 3.2. With the constant $C_{1}$ depending on $\tau$ (but not on $k$ ) the estimate (3.2) follows from the conjugation $u \mapsto v=e^{-i k x} u$ and the unique continuation property for $-\partial_{x}^{2}+W, W \in L^{p}, p>1$. As pointed out in [14], this result is implicit in the paper of Schechter-Simon [21].

To obtain the dependence of constants for large $\langle\tau\rangle$ we first observe that interpolation between the $H^{-1}$ and $L^{2}$ estimates in Lemma 3.3 shows that if $\left(-\left(\partial_{x}+i k\right)^{2}-\tau\right) u=$ $g+f$, then

$$
\|u\|_{L^{2}}+\langle\tau\rangle^{-1 / 2}\left\|\partial_{x} u\right\|_{L^{2}} \leq C\langle\tau\rangle^{-1 / 2}\|g\|_{L^{2}}+C\langle\tau\rangle^{(s-1) / 2}\|f\|_{H^{-s}}+C\|u\|_{L^{2}(\omega)}
$$

for $0 \leq s \leq 1$. As a consequence, if $\left(-\left(\partial_{x}+i k\right)^{2}-\tau\right) u=g-W u$, then

$$
\|u\|_{L^{2}} \leq C\langle\tau\rangle^{-1 / 2}\|g\|_{L^{2}}+C\langle\tau\rangle^{(s-1) / 2}\|W u\|_{H^{-s}}+C\|u\|_{L^{2}(\omega)} .
$$

By Sobolev embeddings, for any $s<1 / 2$, there exists $C>0$ such that for any $u \in$ $H^{s}\left(\mathbb{T}^{1}\right)$,

$$
\|u\|_{L^{2 /(1-2 s)}\left(\mathbb{T}^{1}\right)} \leq C\|u\|_{H^{s}\left(\mathbb{T}^{1}\right)} .
$$

By duality, we deduce $L^{2 /(1+2 s)}\left(\mathbb{T}^{1}\right) \rightarrow H^{-s}\left(\mathbb{T}^{1}\right)$. Choosing $s=1 /(2 p)<1 / 2$, and applying Hölder's inequality we obtain

$$
\begin{aligned}
\|W u\|_{H^{-s}} & \leq C\|W u\|_{L^{2 /(1+2 s)}} \leq C\|W\|_{L^{p}}\|u\|_{L^{2 /(1-2 s)}} \\
& \leq C\|W\|_{L^{p}}\|u\|_{H^{s}} \leq C^{\prime}\|W\|_{L^{p}}\|u\|_{L^{2}}^{1-s}\left(\|u\|_{L^{2}}+\left\|\partial_{x} u\right\|_{L^{2}}\right)^{s} \\
& \leq C^{\prime}\|W\|_{L^{p}}\left(\langle\tau\rangle^{(1+\delta) s^{2} /(2(1-s))}\|u\|_{L^{2}}+\langle\tau\rangle^{-(1+\delta) s / 2}\left\|\partial_{x} u\right\|_{L^{2}}\right) .
\end{aligned}
$$

Combining this with (3.7) yields

$$
\begin{aligned}
\|u\|_{L^{2}}+\langle\tau\rangle^{-1 / 2}\left\|\partial_{x} u\right\|_{L^{2}} \leq C\langle\tau\rangle^{-1 / 2}\|g\|_{L^{2}}+C\|u\|_{L^{2}(\omega)} & \\
& +C_{2}\langle\tau\rangle^{(s-1) / 2}\langle\tau\rangle^{(1+\delta) s^{2} /(2(1-s))}\|u\|_{L^{2}}+C_{3}\langle\tau\rangle^{(s-1) / 2}\langle\tau\rangle^{-(1+\delta) s / 2}\|u\|_{H^{1}} .
\end{aligned}
$$

Since $0<s<1$, taking $\langle\tau\rangle$ large enough allows us to absorb the last term on the righthand side in the left-hand side. The same is true for the third term since

$$
\frac{(1+\delta) s^{2}}{2(1-s)}+\frac{s-1}{2}=\frac{-1+2 s+\delta s^{2}}{1-s},
$$

which is negative for $0<s<1 / 2$ if we choose $\delta$ small enough.

Proof of Proposition 3.1. Let us now show how to pass from the estimate in Proposition 3.2 to an observability result. This was already achieved in [6] in a more general semiclassical setting. For completeness we present a simple version of it here-see [20].

For $\chi \in C_{0}^{\infty}(\mathbb{R})$, put $w=\chi(t) e^{i t P} u_{0}$, which solves

$$
\left(i \partial_{t}+P\right) w=i \chi^{\prime}(t) e^{i t P} u_{0}=v, \quad P:=-\left(\partial_{x}+i k\right)^{2}+W(x) .
$$

Taking Fourier transforms with respect to time, we get

$$
(P-\tau) \widehat{w}(\tau)=\widehat{v}(\tau)
$$


Using the estimate in Proposition 3.2, we write

$$
\|\widehat{w}(\tau)\|_{L^{2}\left(\mathbb{T}^{1}\right)} \leq \frac{C}{1+\sqrt{|\tau|}}\|\widehat{v}(\tau)\|_{L^{2}\left(\mathbb{T}^{1}\right)}+C\|\widehat{w}(\tau)\|_{L^{2}(\omega)} .
$$

Now, taking the $L^{2}$ norm with respect to the $\tau$ variable gives

$$
\begin{aligned}
& \|\widehat{w}(\tau)\|_{L^{2}\left(\mathbb{R}_{\tau} \times \mathbb{T}^{1}\right)} \\
& \quad \leq \frac{C}{1+\sqrt{N}}\|\widehat{v}(\tau)\|_{L^{2}\left(\mathbb{R}_{\tau} \times \mathbb{T}^{1}\right)}+C\|\widehat{w}(\tau)\|_{L^{2}\left(\mathbb{R}_{\tau} \times \omega\right)}+\left(\int_{\mid \tau \leq N}\|\widehat{v}(\tau)\|_{L^{2}\left(\mathbb{T}^{1}\right)}^{2} d \tau\right)^{1 / 2} .
\end{aligned}
$$

From this we notice that

$$
\begin{gathered}
\|\widehat{w}(\tau)\|_{L^{2}\left(\mathbb{R}_{\tau} \times \mathbb{T}^{1}\right)}=\left\|u_{0}\right\|_{L^{2}\left(\mathbb{T}^{1}\right)}\|\chi\|_{L^{2}(\mathbb{R})}, \quad\|\widehat{v}(\tau)\|_{L^{2}\left(\mathbb{R}_{\tau} \times \mathbb{T}^{1}\right)}=\left\|u_{0}\right\|_{L^{2}\left(\mathbb{T}^{1}\right)}\left\|\chi^{\prime}\right\|_{L^{2}(\mathbb{R})}, \\
\|\widehat{w}(\tau)\|_{L^{2}\left(\mathbb{R}_{\tau} \times \omega\right)}=\left\|\chi(t) e^{i t P} u_{0}\right\|_{L^{2}\left(\mathbb{R}_{t} \times \mathbb{T}^{1}\right)} .
\end{gathered}
$$

Hence we deduce that if

$$
\frac{C\left\|\chi^{\prime}\right\|_{L^{2}}}{\|\chi\|_{L^{2}}(1+\sqrt{N})} \leq \frac{1}{2}
$$

then

$$
\left\|u_{0}\right\|_{L_{x}^{2}} \leq C^{\prime}\left\|\chi(t) e^{i t P} u_{0}\right\|_{L^{2}\left(\mathbb{R}_{t} \times \mathbb{T}_{x}^{1}\right)}+C^{\prime}\left(\int_{|\tau| \leq N}\|\widehat{v}(\tau)\|_{L_{x}^{2}}^{2} d \tau\right)^{1 / 2} .
$$

To understand the last term on the right-hand side we define Sobolev norms associated to $P$. Let $\left\{\varphi_{n}\right\}_{n=1}^{\infty}$ be an orthonormal basis of $L^{2}\left(\mathbb{T}^{1}\right)$ consisting of eigenfuctions of $P$. We then put

$$
\|u\|_{H_{P}^{k}}^{2}:=\sum_{j=1}^{\infty}\left\langle\lambda_{n}\right\rangle^{2 k}\left|u_{n}\right|^{2}, \quad P \varphi_{n}=\lambda_{n} \varphi_{n}, \quad u_{n}:=\left\langle u, \varphi_{n}\right\rangle .
$$

In this notation $w=\chi(t) \sum_{n} u_{n} e^{-i t \lambda_{n}} \varphi_{n}$, and

$$
\widehat{v}(\tau)=\sum_{n} \widehat{\chi^{\prime}}\left(\tau-\lambda_{n}\right) u_{n} \varphi_{n}
$$

Hence

$$
\begin{aligned}
\int_{0}^{N}\|\widehat{v}(\tau)\|_{L_{x}^{2}}^{2} d \tau & =\sum_{n=1}^{\infty}\left|u_{n}\right|^{2} \int_{0}^{N}\left|\left(\tau-\lambda_{n}\right) \hat{\chi}\left(\tau-\lambda_{n}\right)\right|^{2} d \tau \\
& =\sum_{n=1}^{\infty}\left|u_{n}\right|^{2} \int_{0}^{N} \mathcal{O}\left(\left\langle\tau-\lambda_{n}\right\rangle^{-\infty}\right) d \tau \\
& \leq C_{N, M} \sum_{n=1}^{\infty}\left\langle\lambda_{n}\right\rangle^{-M}\left|u_{n}\right|^{2}=C_{N, M}\|u\|_{H_{P}^{-M}}^{2}
\end{aligned}
$$

for any $M$. Taking $M=2$ and combining this with (3.8) we obtain

$$
\left\|u_{0}\right\|_{L^{2}\left(\mathbb{T}^{1}\right)} \leq C\left\|\chi(t) e^{i t P} u_{0}\right\|_{L^{2}\left(\mathbb{R}_{t} \times \omega\right)}+C\left\|u_{0}\right\|_{H_{P}^{-2}}
$$


To complete the proof, it remains to eliminate the last term on the right-hand side of (3.9). For this, we apply the now classical uniqueness-compactness argument of BardosLebeau-Rauch [2] (see also [8, §4]) or the direct argument presented in the Appendix. We note that both approaches rely on the unique continuation property of $-\left(\partial_{x}+i k\right)^{2}+W(x)$, $W \in L^{p}\left(\mathbb{T}^{1}\right), p>1$. Notice also that in this argument, to get the independence of the constant from $k \in[0,1]$, it is enough to use the compactness of $[0,1]$.

For later use we also record the following approximation result:

Proposition 3.4. Assume that the sequence of potentials $W_{j}$ converges to $W$ in $L^{p}\left(\mathbb{T}^{1}\right)$, $p \geq 2$. Then there exists $K_{0}>0$ such that for any $k \in \mathbb{R}$ and $u \in L^{2}\left(\mathbb{T}^{1}\right)$, and any $j \in \mathbb{N}$,

$$
\|u\|_{L^{2}\left(\mathbb{T}^{1}\right)}^{2} \leq K_{0} \int_{0}^{T}\left\|e^{i t\left(\left(\partial_{x}+i k\right)^{2}-W_{j}\right)} v\right\|_{L^{2}(\omega)}^{2} d t .
$$

Proof. The conclusion follows from Proposition 3.1 by a simple perturbation argument. Put $P=-\left(\partial_{x}+i k\right)^{2}+W$ and $P_{j}=-\left(\partial_{x}^{2}+i k\right)^{2}+W_{j}$. Then, according to the Duhamel formula, we have

$$
e^{-i t P} v=e^{-i t P_{j}} v+\frac{1}{i} \int_{0}^{t} e^{-i(t-s) P_{j}}\left(W-W_{j}\right) e^{-i s P} v d s,
$$

and consequently, according to (2.3), we obtain

$$
\begin{aligned}
\left\|e^{-i t P} v-e^{-i t P_{j}} v\right\|_{L^{\infty}\left((0, T) ; L^{2}\left(\mathbb{T}^{1}\right)\right)} & \leq C\left\|\left(W-W_{j}\right) e^{-i s P} v\right\|_{L^{1}\left((0, T) ; L^{2}\left(\mathbb{T}^{1}\right)\right)} \\
& \leq C \sqrt{T}\left\|W-W_{j}\right\|_{L^{2}\left(\mathbb{T}^{1}\right)}\left\|e^{-i s P} v\right\|_{L^{\infty}\left(\mathbb{T}_{x}^{1} ; L^{2}(0, T)\right)} \\
& \leq C \sqrt{T}\left\|W-W_{j}\right\|_{L^{2}\left(\mathbb{T}^{1}\right)}\|v\|_{L^{2}\left(\mathbb{T}^{1}\right)} .
\end{aligned}
$$

According to (3.1) we have

$$
\begin{aligned}
\|v\|_{L^{2}\left(\mathbb{T}^{1}\right)}^{2} & \leq K_{0} \int_{0}^{T}\left\|e^{-i t P} v\right\|_{L^{2}(\omega)}^{2} d t \\
& \leq 2 K_{0} \int_{0}^{T}\left\|e^{-i t P_{j}} v\right\|_{L^{2}(\omega)}^{2} d t+2 C^{2} T\left\|W-W_{j}\right\|_{L^{2}\left(\mathbb{T}^{1}\right)}^{2}\|v\|_{L^{2}\left(\mathbb{T}^{1}\right)}^{2},
\end{aligned}
$$

which implies (3.10) if $\left\|W-W_{j}\right\|_{L^{2}\left(\mathbb{T}^{1}\right)}$ is small enough.

\section{Semiclassical observation estimates in dimension 2}

We revisit and refine the arguments of [8]. The key point in our analysis will be the following variant of [8, Proposition 3.1]. The key difference is that now the main constant is determined in terms of the geometry of the problem and the potential $V$. 
Proposition 4.1. Suppose that $V_{j} \in C^{\infty}\left(\mathbb{T}^{2} ; \mathbb{R}\right)$ converges to $V$ in the $L^{2}\left(\mathbb{T}^{2}\right)$ topology. Let $\chi \in C_{0}^{\infty}(-1,1)$ be equal to 1 near 0 , and define

$$
\Pi_{h, \rho, j} u_{0}:=\chi\left(\frac{h^{2}\left(-\Delta+V_{j}\right)-1}{\rho}\right) u_{0}, \quad \rho>0 .
$$

Then for any non-empty open subset $\Omega$ of $\mathbb{T}^{2}$ and $T>0$, there exists a constant $K>0$ such that for any $j$ there exist $\rho_{j}, h_{0, j}>0$ such that for any $0<h<h_{0, j}$ and $u_{0} \in$ $L^{2}\left(\mathbb{T}^{2}\right)$,

$$
\left\|\Pi_{h, \rho_{j}, j} u_{0}\right\|_{L^{2}}^{2} \leq K \int_{0}^{T}\left\|e^{-i t\left(-\Delta+V_{j}\right)} \Pi_{h, \rho_{j}, j} u_{0}\right\|_{L^{2}(\Omega)}^{2} d t .
$$

In the proof we argue by contradiction. We first observe that if the estimate (4.1) is true for some $\rho>0$, then it is true for all $0<\rho^{\prime}<\rho$. As a consequence, if (4.1) were false then for any $j$, there would exist sequences

$$
\begin{gathered}
h_{n, j} \rightarrow 0, \quad \rho_{n, j} \rightarrow 0, \quad u_{0, n, j}=\Pi_{h_{n, j}, \rho_{n, j}, j} v_{0, n, j} \in L^{2}, \\
i \partial_{t} u_{n, j}(t, z)=\left(-\Delta+V_{j}(z)\right) u_{n, j}(t, z), \quad u_{n, j}(0, z)=u_{0, n, j}(z),
\end{gathered}
$$

such that

$$
1=\left\|u_{0, n, j}\right\|_{L^{2}}^{2}, \quad \int_{0}^{T}\left\|u_{n, j}(t, \cdot)\right\|_{L^{2}(\Omega)}^{2} d t \leq \frac{1}{K} .
$$

Each sequence $n \mapsto u_{n, j}$ is bounded in $L_{\text {loc }}^{2}\left(\mathbb{R} \times \mathbb{T}^{2}\right)$ and consequently, after possibly extracting a subsequence, there exists a semiclassical defect measure $\mu_{j}$ on $\mathbb{R}_{t} \times T^{*} \mathbb{T}_{z}^{2}$ such that for any function $\varphi \in C_{0}^{0}\left(\mathbb{R}_{t}\right)$ and any $a \in C_{0}^{\infty}\left(T^{*} \mathbb{T}_{z}^{2}\right)$, we have

$$
\left\langle\mu_{j}, \varphi(t) a(z, \zeta)\right\rangle=\lim _{n \rightarrow \infty} \int_{\mathbb{R}_{t} \times \mathbb{T}^{2}} \varphi(t)\left(a\left(z, h_{n, j} D_{z}\right) u_{n, j}\right)(t, z) \bar{u}_{n, j}(t, z) d t d z .
$$

Furthermore, standard arguments ${ }^{\ddagger}$ show that:

- We have

$$
\mu_{j}\left(\left(t_{0}, t_{1}\right) \times T^{*} \mathbb{T}_{z}^{2}\right)=t_{1}-t_{0} .
$$

- The measure $\mu_{j}$ on $\mathbb{R}_{t} \times T^{*}\left(\mathbb{T}^{2}\right)$ is supported in the set

$$
\Sigma:=\left\{(t, z, \zeta) \in \mathbb{R}_{t} \times \mathbb{T}_{z}^{2} \times \mathbb{R}_{\zeta}^{2} ;|\zeta|=1\right\}
$$

and is invariant under the action of the geodesic flow:

$$
\xi \cdot \nabla_{x}\left(\mu_{j}\right)=0 .
$$

- The mass of the measure on $\Omega$ is bounded:

$$
\mu_{j}\left((0, T) \times T^{*} \Omega\right) \leq 1 / K .
$$

\footnotetext{
$\ddagger$ See [1] for a review of recent results about measures used for the Schrödinger equation.
} 
We are going to show that a proper choice of the constant $K$ above contradicts (4.3). When no confusion is likely to occur we will drop the index $j$ for conciseness.

We start by decomposing $\Sigma$ into its rational and irrational parts. For that we identify $\mathbb{T}^{2} \simeq[0, A)_{x} \times[0, B)_{y}$ where $A, B \in \mathbb{R} \backslash\{0\}$, and define

$$
\Sigma_{\mathbb{Q}}:=\Sigma \cap\left\{\left(t, z, \frac{(A p, B q)}{\sqrt{A^{2} p^{2}+B^{2} q^{2}}}\right) ; p, q \in \mathbb{Z}, \operatorname{gcd}(p, q)=1\right\} .
$$

The flow on $\Sigma_{\mathbb{Q}}$ is periodic. Its complement is the set of irrational points,

$$
\Sigma_{\mathbb{R} \backslash \mathbb{Q}}:=\Sigma \backslash \Sigma_{\mathbb{Q}},
$$

and it also invariant under the flow.

\subsection{The irrational directions}

For simplicity we assume here that $A=B=2 \pi$, that is, $\mathbb{T}^{2}=\mathbb{T}^{1} \times \mathbb{T}^{1}$, as the argument is the same as in the general case.

Let us first define $\mu_{\mathbb{R} \backslash \mathbb{Q}}$ to be the restriction of the measure $\mu$ to $\Sigma_{\mathbb{R} \backslash \mathbb{Q}}$. Since $\mu$ is invariant, for any open set $\Omega \subset \mathbb{T}^{2}$ and any $s \in \mathbb{R}$,

$$
\mu_{\mathbb{R} \backslash \mathbb{Q}}\left(\left(t_{1}, t_{2}\right) \times \Omega \times \mathbb{R}^{2}\right)=\mu_{\mathbb{R} \backslash \mathbb{Q}}\left(\left(t_{1}, t_{2}\right) \times \Phi_{s}\left(\Omega \times \mathbb{R}^{2}\right)\right)
$$

where the flow $\Phi_{s}$ is defined by $\Phi_{s}(z, \zeta)=(z+s \zeta, \zeta)$. As a consequence, for any $S>0$,

$$
\begin{aligned}
\mu_{\mathbb{R} \backslash \mathbb{Q}}\left(\left(t_{1}, t_{2}\right) \times \Omega \times \mathbb{R}^{2}\right) & =\frac{1}{S} \int_{0}^{S} \mu_{\mathbb{R} \backslash \mathbb{Q}}\left(\left(t_{1}, t_{2}\right) \times \Phi_{S}\left(\Omega \times \mathbb{R}^{2}\right)\right) d s \\
& =\int \mathbb{1}_{t \in\left(t_{1}, t_{2}\right)} \times \frac{1}{S} \int_{0}^{S} \mathbb{1}_{(z, \zeta) \in \Phi_{s}\left(\Omega \times \mathbb{R}^{2}\right)} d s d \mu_{\mathbb{R} \backslash \mathbb{Q}} .
\end{aligned}
$$

The equidistribution theorem shows that for any $(z, \zeta)$ in the support of $\mu_{\mathbb{R} \backslash \mathbb{Q}}$,

$$
\lim _{S \rightarrow \infty} \frac{1}{S} \int_{0}^{S} \mathbb{1}_{(z, \zeta) \in \Phi_{s}\left(\Omega \times \mathbb{R}^{2}\right)} d s=\frac{\operatorname{vol}(\Omega)}{\operatorname{vol}\left(\mathbb{T}^{2}\right)}
$$

Hence the dominated convergence theorem and (4.3) show that

$$
\mu_{\mathbb{R} \backslash \mathbb{Q}}\left(\left(t_{1}, t_{2}\right) \times \Omega \times \mathbb{R}^{2}\right)=\frac{\operatorname{vol}(\Omega)}{\operatorname{vol}\left(\mathbb{T}^{2}\right)} \mu_{\mathbb{R} \backslash \mathbb{Q}}\left(\left(t_{1}, t_{2}\right) \times \mathbb{T}^{2} \times \mathbb{R}^{2}\right)
$$

\subsection{Dense rational directions}

We now consider the restriction of the measure $\mu$ to the set of rational directions, $\Sigma_{\mathbb{Q}}$. We first consider the case of $p / q$ for which $p^{2}+q^{2}$ is large (we again assume that $A=B=1$ as the general argument is the same). In some sense that corresponds to being close to the irrational case. 
Lemma 4.2. For any open set $\Omega$, there exist $N \in \mathbb{N}$ and $\delta>0$ such that for any $(p, q) \in \mathbb{Z}^{2}$ with $\operatorname{gcd}(p, q)=1$ and $\sqrt{p^{2}+q^{2}} \geq N$,

$$
\liminf _{S \rightarrow \infty} \frac{1}{S} \int_{0}^{S} \mathbb{1}_{(z, \zeta) \in \Phi_{S}\left(\Omega \times \mathbb{R}^{2}\right)} d s \geq \delta, \quad \zeta=\frac{(p, q)}{\sqrt{p^{2}+q^{2}}} .
$$

Proof. For any $z_{0}=\left(x_{0}, y_{0}\right) \in \Omega$ choose $N>4 \pi / \epsilon$ where $B\left(z_{0}, 2 \epsilon\right) \subset \Omega$. Assume that $p \geq N / 2>2 \pi / \epsilon$ and that $p \geq q$ (the case of $q \leq p$ is similar). Put

$$
s_{k}:=\frac{\sqrt{p^{2}+q^{2}}}{p}\left(2 k \pi-x_{0}\right), \quad k=0, \ldots, p-1 .
$$

Since $p$ and $q$ are coprime, $q$ is a generator of the group $\mathbb{Z} / p \mathbb{Z}$. Consequently, the points

$$
Y_{k}=\frac{s_{k}}{\sqrt{p^{2}+q^{2}}} q-y_{0} \in \mathbb{T}^{1}
$$

are at distance exactly $2 \pi / p$ from each other. (Here and below, addition on $\mathbb{T}^{1}$ is meant $\bmod 2 \pi \mathbb{Z}$.) We conclude that for any $z \in \mathbb{T}^{1}$ there exists

$$
J_{z} \subset\{0, \ldots, p-1\}, \quad\left|J_{z}\right|=[\epsilon p / \pi], \quad \text { such that } \quad\left|y+Y_{k}-y_{0}\right| \leq \epsilon \text { for } k \in J_{z} .
$$

Since the flow is given by

$$
\Phi_{-s}\left((x, y), \frac{(p, q)}{\sqrt{p^{2}+q^{2}}}\right)=\left((x, y)-\frac{s}{\sqrt{p^{2}+q^{2}}}(p, q), \frac{(p, q)}{\sqrt{p^{2}+q^{2}}}\right),
$$

for any $k \in J$, we have $\Phi_{-s_{k}}\left(z,(p, q) / \sqrt{p^{2}+q^{2}}\right) \in B\left(z_{0}, \epsilon\right) \times \mathbb{R}^{2}$. Since $2 \pi / p<\epsilon$, we also find that for $\left|s-s_{k}\right|<\epsilon$,

$$
\Phi_{-s}\left(z, \frac{(p, q)}{\sqrt{p^{2}+q^{2}}}\right) \in B\left(z_{0}, 2 \epsilon\right) \times \mathbb{R}^{2} \subset \Omega \times \mathbb{R}^{2} .
$$

Hence, using the assumption that $q \leq p$,

$\int_{0}^{2 \pi \sqrt{p^{2}+q^{2}}} \mathbb{1}_{\Phi_{-s}(z, \zeta) \in \Omega \times \mathbb{R}^{2}} d s \geq[\epsilon p / \pi] \epsilon>2 \pi \sqrt{p^{2}+q^{2}} \delta, \quad \zeta=(p, q) / \sqrt{p^{2}+q^{2}}$,

for some $\delta>0$. Since the evolution of $(z, \zeta)$ is periodic with period $2 \pi \sqrt{p^{2}+q^{2}}$, the lemma follows.

Let us now fix $N$ as in Lemma 4.2 and let $\mu_{\mathbb{Q}, N}$ be the restriction of $\mu_{\mathbb{Q}}$ to rational directions satisfying $\sqrt{p^{2}+q^{2}} \geq N$. As in the study of the irrational directions, Lemma 4.2 and Fatou's Lemma imply

$$
\mu_{\mathbb{Q}, N}\left(\left(t_{1}, t_{2}\right) \times \Omega \times \mathbb{R}^{2}\right) \geq \delta \mu_{\mathbb{Q}, N}\left(\left(t_{1}, t_{2}\right) \times \mathbb{T}^{2} \times \mathbb{R}^{2}\right) .
$$




\subsection{Isolated rational directions}

This section is closest to the arguments of $[8, \S 3]$. We allow here existence of points in $\Sigma_{\mathbb{Q}}$ whose evolution misses $\Omega$ altogether. The contradiction is derived from that assumption. It is now important to keep $A$ and $B$ arbitrary, $\mathbb{T}^{2}=\mathbb{R}^{2} / A \mathbb{Z} \times B \mathbb{Z}$. The constraints on the constant $K$ will not be only geometric as in $\S \S 4.1,4.2$, but will also involve the limit potential $V$. Hence we return to the notation of (4.2) and keep the index $j$.
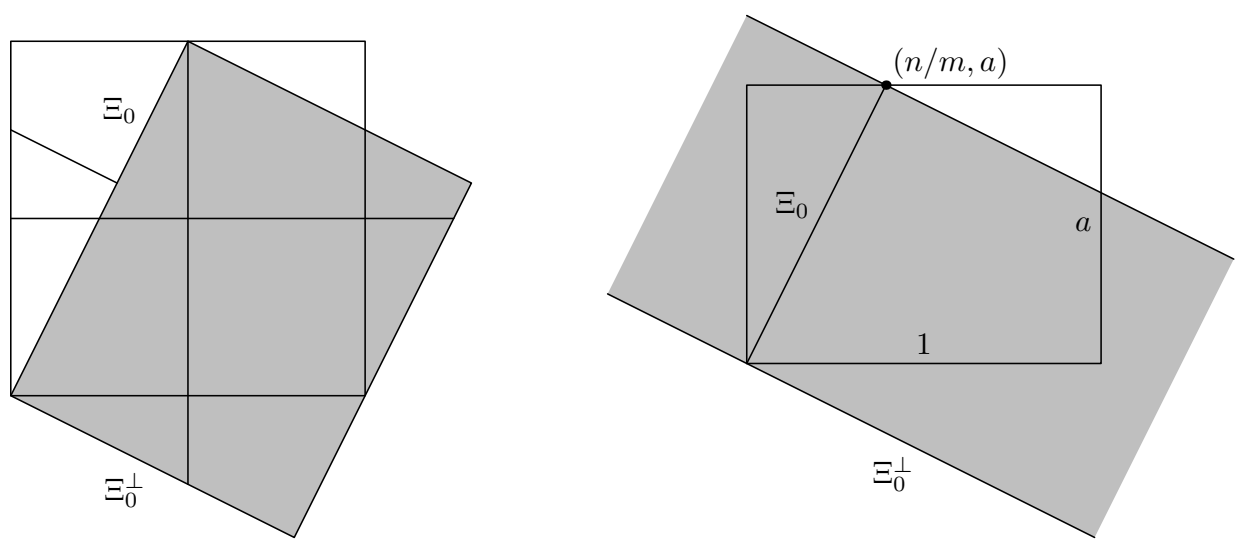

Fig. 2. Left: a rectangle, $R$, covering a rational torus $\mathbb{T}^{2}$. In that case we obtain a periodic solution on $R$. Right: the irrational case; the strip with sides $m \Xi_{0} \times \mathbb{R}_{0}^{\perp}, \Xi_{0}=(n / m, a)$ (not normalized to have norm one) also covers the torus $[0,1] \times[0, a]$. Periodic functions are pulled back to functions satisfying (4.10). This figure is borrowed from [8].

We consider the restriction of the measure $\mu$ to any of the finitely many isolated rational directions:

$$
\Xi_{0}=\frac{(A p, B q)}{\sqrt{A^{2} p^{2}+B^{2} q^{2}}}, \quad \sqrt{p^{2}+q^{2}} \leq N .
$$

We first recall the following simple result [8, Lemma 2.7] (see Fig. 2 for an illustration).

Lemma 4.3. Suppose that $\Xi_{0}$ is given by (4.8) and

$$
F:(x, y) \mapsto z=F(x, y)=x \Xi_{0}^{\perp}+y \Xi_{0}, \quad \Xi_{0}^{\perp}=\frac{1}{\sqrt{A^{2} p^{2}+B^{2} q^{2}}}(B q,-A p) .
$$

If $u=u(z)$ is periodic with respect to $A \mathbb{Z} \times B \mathbb{Z}$ then

$$
F^{*} u(x+k a, y+\ell b)=F^{*} u(x, y-k \gamma), \quad k, \ell \in \mathbb{Z},(x, y) \in \mathbb{R}^{2},
$$

where, for any fixed $p, q \in \mathbb{Z}$,

$$
a=\frac{-\left(q^{2}+p^{2}\right) A B}{\sqrt{A^{2} p^{2}+B^{2} q^{2}}}, \quad b=\sqrt{A^{2} p^{2}+B^{2} q^{2}}, \quad \gamma=-\frac{p q\left(B^{2}-A^{2}\right)}{\sqrt{A^{2} p^{2}+B^{2} q^{2}}} .
$$


When $B / A=r / s \in \mathbb{Q}$ then

$$
F^{*} u(x+k \tilde{a}, y+\ell b)=F^{*} u(x, y), \quad k, \ell \in \mathbb{Z},(x, y) \in \mathbb{R}^{2},
$$

for $\widetilde{a}=\left(p^{2} s^{2}+q^{2} r^{2}\right) a$.

Indeed, with

$$
\mathcal{A}=\frac{1}{\sqrt{A^{2} p^{2}+B^{2} q^{2}}}\left(\begin{array}{cc}
q B & p A \\
-p A & q B
\end{array}\right)
$$

we have

$$
\mathcal{A}\left(\begin{array}{l}
0 \\
b
\end{array}\right)=\left(\begin{array}{c}
-p A \\
q B
\end{array}\right), \quad \mathcal{A}\left(\begin{array}{l}
a \\
\gamma
\end{array}\right)=\left(\begin{array}{c}
-q A \\
p B
\end{array}\right),
$$

which implies

$$
u\left(\mathcal{A}\left(\begin{array}{c}
x+k a \\
y+k \gamma+l b
\end{array}\right)\right)=u\left(\mathcal{A}\left(\begin{array}{l}
x \\
y
\end{array}\right)\right) .
$$

We now identify $u_{n, j}$ with $F^{*} u_{n, j}$, and consider the Schrödinger equation on the strip $R=\mathbb{R}_{x} \times[0, b]_{y}$ (or the rectangle $R=[0, a]_{x} \times[0, b]_{y}$ in the case when $A / B \in \mathbb{Q}$ ). In this coordinate system, $\Xi_{0}=(0,1)$.

Choosing a function $\chi \in C_{0}^{\infty}\left(\mathbb{R}^{2}\right)$ equal to 1 near $(0,0)$ we define, for $\epsilon>0$,

$$
\chi_{\epsilon}:=\chi(((\eta, \zeta)-(0,1)) / \epsilon), \quad \eta, \zeta \in \mathbb{R},
$$

and

$$
u_{n, j, \epsilon}(x, y)=\chi_{\epsilon}\left(h_{n, j} D_{x}\right) u_{n, j}
$$

We denote by $\mu_{j, \epsilon}$ the semiclassical measure of the sequence $\left(u_{n, j, \epsilon}\right)_{n \in \mathbb{N}}(j, \epsilon$ are parameters). Since $\mu_{j, \epsilon}=\left(\chi_{\epsilon}(\zeta)\right)^{2} \mu_{j}$ (where we skipped the pull-back by $F$ ), we have

$$
\lim _{\epsilon \rightarrow 0+} \mu_{j, \epsilon}=\left.\mu_{j}\right|_{\{(t, z, \zeta) ; \zeta=(0,1)\}} .
$$

We now recall the following normal-form result given in [8, Proposition 2.3 and Corollary 2.4]:

Proposition 4.4. Suppose that $F: \mathbb{R}^{2} \rightarrow \mathbb{R}^{2}$ is given by (4.9) and that $V \in C^{\infty}\left(\mathbb{R}^{2}\right)$ is periodic with respect to $A \mathbb{Z} \times B \mathbb{Z}$. Let $a, b$ and $\gamma$ be as in (4.10). Let $\chi \in C_{0}^{\infty}\left(\mathbb{R}^{2}\right)$ be equal to 0 in a neighbourhood of $\eta=0$. Suppose that $V_{j}(x, y) \in C^{\infty}\left(\mathbb{T}^{1} \times \mathbb{T}^{1}\right)$. Then there exist operators

$$
Q_{j}\left(x, y, h D_{y}\right) \in C^{\infty}(\mathbb{R}) \otimes \Psi^{0}(\mathbb{R}), \quad R_{j}\left(x, y, h D_{x}, h D_{y}\right) \in \Psi^{0}\left(\mathbb{R}^{2}\right),
$$

(where $\Psi^{0}$ denotes the space of semiclassical pseudodifferential operators of order 0 ) such that $\left(F^{-1}\right)^{*} Q F^{*}$ and $\left(F^{-1}\right)^{*} R F^{*}$ preserve $A \mathbb{Z} \times B \mathbb{Z}$ periodicity, and

$$
\begin{array}{r}
\left(I+h Q_{j}\right)\left(D_{y}^{2}+F^{*} V_{j}(x, y)\right) \chi\left(h D_{x}, h D_{y}\right) \\
=\left(D_{y}^{2}+W_{j}(x)\right)\left(I+h Q_{j}\right) \chi\left(h D_{x}, h D_{y}\right)+h R_{j},
\end{array}
$$

where $W_{j}(x)=(1 / b) \int_{0}^{b} F^{*} V_{j}(x, y) d y$ satisfies $W_{j}(x+a)=W_{j}(x)$. 
Moreover, there exist operators $P_{j}=P_{j}\left(x, y, h D_{x}, h D_{y}\right) \in \Psi^{0}\left(\mathbb{R}^{2}\right)$ such that (with properties as above)

$$
\begin{gathered}
\left(I+h Q_{j}\right)\left(D_{x}^{2}+D_{y}^{2}+F^{*} V_{j}(x, y)\right) \chi\left(h D_{x}, h D_{y}\right) \\
=\left(\left(D_{x}^{2}+D_{y}^{2}+W_{j}(x)\right)\left(I+h Q_{j}\right)+P_{j}\right) \chi\left(h D_{x}, h D_{y}\right)+h R_{j}, \\
P_{j}(x, y, x, \eta)=\frac{2}{i} \xi \partial_{x} q_{j}(x, y, \eta) \tilde{\chi}_{\epsilon}(\xi, \eta), \quad q_{j}=\sigma\left(Q_{j}\right),
\end{gathered}
$$

where $\tilde{\chi} \in C_{0}^{\infty}\left(\mathbb{R}^{2}\right)$ is equal to 1 on the support of $\chi$.

Using Proposition 4.4 we define

$$
v_{n, j, \epsilon}=\left(1+h Q_{j}\right) u_{n, j, \epsilon}, \quad h=h_{n, j} .
$$

Since the operator $Q_{j}$ is bounded on $L^{2}$, the semiclassical defect measures associated to $v_{n, j, \epsilon}$ and $u_{n, j, \epsilon}$ are equal. We now consider the time dependent Schrödinger equation satisfied by $v_{n, j, \epsilon}$. With

$$
\begin{aligned}
Q_{n, j} & :=Q_{j}\left(x, y, h_{n, j} D_{y}\right), \quad R_{n, j}:=R\left(x, y, h_{n, j} D_{x}, h_{n, j} D_{y}\right), \\
P_{n, j} & :=P_{j}\left(x, y, h_{n, j} D_{x}, h_{n, j} D_{y}\right),
\end{aligned}
$$

given in Proposition 4.4 and $\chi_{n, j, \epsilon}:=\chi\left(h_{n, j} D_{z}\right)$, we have

$$
\begin{aligned}
& \left(i \partial_{t}+\Delta-W_{j}(x)\right) v_{n, j} \\
& =\left(I+h_{n, j} Q_{n, j}\right)\left(i \partial_{t}+\Delta-V_{j}(x, y)\right) \chi_{n, j, \epsilon} u_{n, j}-P_{n, j} \chi_{n, j, \epsilon} u_{n, j}-h_{n, j} R_{n, j, \epsilon} u_{n, j} \\
& =-P_{n, j} \chi_{n, j, \epsilon} u_{n, j}+\left[V, \chi_{n, j, \epsilon}\right] u_{n, j}+o_{L^{2}}(1)=-P_{n, j} \chi_{n, j, \epsilon} u_{n, j}+o_{L_{x, y}^{2}}(1) .
\end{aligned}
$$

We also recall that according to (4.14), on the support of $\mu_{j, \epsilon}$, the symbol of the operator $W$ is smaller than $C \epsilon$. This implies that

$$
\left(i \partial_{t}+\Delta-W_{j}(x)\right) v_{n, j, \epsilon}=f_{n, j, \epsilon}
$$

with

$$
\limsup _{n \rightarrow \infty}\left\|f_{n, j, \epsilon}\right\|_{L^{2}\left((0, T) \times \mathbb{T}^{2}\right)}^{2}=\left\langle\mu_{j, \epsilon},\left|P_{n, j}\right|^{2}\right\rangle \leq C_{j} \epsilon^{2} .
$$

The simple observation that

$$
e^{i t\left(\partial_{y}^{2}+\partial_{x}^{2}-W_{j}(x)\right)}=e^{i t \partial_{y}^{2}} e^{i t\left(\partial_{x}^{2}-W_{j}(x)\right)}
$$

shows that we can write

$$
v_{n, j, \epsilon}(t, x, y)=\sum_{k \in \mathbb{Z}} e^{-i\left(t k^{2}+k y\right)} v_{n, j, \epsilon, k}(t, x), \quad f_{n, j, \epsilon}(t, x, y)=\sum_{k \in \mathbb{Z}} e^{-i k y} f_{n, j, \epsilon, k}(t, x),
$$

where

$$
\left(i \partial_{t}+\partial_{x}^{2}-W_{j}(x)\right) v_{n, j, \epsilon, k}=f_{n, j, \epsilon, k}
$$

and the coefficients satisfy the Floquet condition (see [8, proof of Proposition 2.2])

$$
\begin{aligned}
& v_{n, j, \epsilon, k}(t, x+a)=e^{2 \pi i \gamma k / b} v_{n, j, \epsilon, k}(t, x)=e^{2 \pi i \gamma_{k}} v_{n, j, \epsilon, k}(t, x), \\
& f_{n, j, \epsilon, k}(t, x+a)=e^{2 \pi i \gamma_{k}} f_{n, j, \epsilon, k}(t, x), \quad \gamma_{k}:=\gamma k / b=[\gamma k / b] \in[0,1) .
\end{aligned}
$$


Since $W_{j}(x+a)=W_{j}(x)$ and

$$
\begin{aligned}
\left\|W-W_{j}\right\|_{L^{2}\left([0, a]_{x}\right)}^{2} & =\int_{0}^{a}\left(\frac{1}{b} \int_{0}^{b} \int\left(F^{*} V(x, y)-F^{*} V_{j}(x, y)\right) d y\right)^{2} d x \\
& \leq\left\|F^{*}\left(V-V_{j}\right)\right\|_{L^{2}\left([0, a]_{x} \times[0, b]_{y}\right)}^{2} \\
& \leq C_{\Xi_{0}}\left\|V-V_{j}\right\|_{L^{2}\left(\mathbb{T}^{2}\right)} \rightarrow 0, \quad j \rightarrow \infty,
\end{aligned}
$$

we can apply Proposition 3.4 to $u_{n, j, \epsilon, k}(t, x)=e^{-2 i \pi \gamma k x /(a b)} v_{n, j, \epsilon, k}(t, x)$, which is periodic on the torus $\mathbb{R} / a \mathbb{Z}$. For that we fix a domain $\omega \subset[0, a]_{x}$ such that for any $x \in \bar{\omega}$, the line $\{x\} \times[0, b]_{y}$ encounters $\Omega$. The estimate (3.10) gives the following non-geometric estimate (it is here that the dependence on the potential enters):

$$
\begin{gathered}
\left\|v_{n, j, \epsilon, k}\right\|_{L^{\infty}\left((0, T) ; L^{2}\left([0, a]_{x}\right)\right)}^{2} \leq 2\left\|\left.v_{n, j, \epsilon, k}\right|_{t=0}\right\|_{L^{2}\left([0, a]_{x}\right)}+2\left\|f_{n, j, \epsilon, k}\right\|_{L^{1}\left((0, T) ; L^{2}\left([0, a]_{x}\right)\right)}^{2} \\
\leq K_{0} \int_{0}^{T}\left\|\left.e^{i t\left(\partial_{x}^{2}-W_{j}(x)\right)} v_{n, j, \epsilon, k}\right|_{t=0}\right\|_{L^{2}(\omega)}^{2} d t+C\left\|f_{n, j, \epsilon, k}\right\|_{L^{2}\left((0, T) \times[0, a]_{x}\right)}^{2} \\
\leq K_{0} \int_{0}^{T}\left\|v_{n, j, \epsilon, k}\right\|_{L^{2}(\omega)}^{2} d t+C\left\|f_{n, j, \epsilon, k}\right\|_{L^{2}\left((0, T) \times[0, a]_{x}\right)}^{2} .
\end{gathered}
$$

Summing over $k \in \mathbb{Z}$ gives

$$
\begin{aligned}
& \left\|v_{n, j, \epsilon}\right\|_{L^{\infty}\left((0, T) ; L^{2}\left([0, a]_{x} \times[0, b]_{y}\right)\right.}^{2} \\
& \quad \leq K_{0} \int_{0}^{T}\left\|\left.v_{n, j, \epsilon}\right|_{t=0}\right\|_{L^{2}(\omega)}^{2} d t+C\left\|f_{n, j, \epsilon}\right\|_{L^{2}\left((0, T) \times[0, a]_{x}\right)}^{2} .
\end{aligned}
$$

Taking first the limit as $n \rightarrow \infty$, we obtain, according to (4.18),

$$
\mu_{j, \epsilon}\left((0, T) \times\left([0, a] \times[0, b]_{y}\right) \times \mathbb{R}^{2}\right) \leq K_{0} \mu_{j, \epsilon}\left((0, T) \times \omega \times[0, b]_{y} \times \mathbb{R}^{2}\right)+C_{j} \epsilon .
$$

Then taking the limit as $\epsilon \rightarrow 0$, we conclude that, according to (4.11),

$\mu_{j}\left((0, T) \times\left([0, a]_{x} \times[0, b]_{y}\right) \times\{(0,1)\}\right) \leq K_{0} \mu_{j}\left((0, T) \times \omega \times[0, b]_{y} \times\{(0,1)\}\right)$.

Since any vertical line over $\bar{\omega}$ encounters the open set $\Omega$, we have

$$
\min _{x \in \bar{\omega}} \int_{\Omega \cap\left(\{x\} \times[0, b]_{y}\right)} d y>\delta_{0}>0 .
$$

This and the invariance of the measure under the flow (which is now just the translation in the $y$ direction) imply that

$$
\mu_{j}\left((0, T) \times \omega \times[0, b]_{y} \times\{(0,1)\}\right) \leq \delta_{0} \mu_{j}((0, T) \times \Omega \times\{(0,1)\}) .
$$

Combining this with (4.19) we find that there exists a constant $K_{(0,1)}$, independent of $j$, such that

$$
\mu_{j}\left((0, T) \times\left([0, a]_{x} \times[0, b]_{y}\right) \times\{(0,1)\}\right) \leq K_{(0,1)} \mu_{j}((0, T) \times \Omega \times\{(0,1)\}) .
$$


Returning to an arbitrary rational direction

$$
\zeta_{p, q}=\frac{(p, q)}{\sqrt{A^{2} p^{2}+B^{2} q^{2}}}, \quad \sqrt{p^{2}+q^{2}} \leq N,
$$

we conclude that there exists a constant $K_{p, q}$ such that

$$
\mu_{j}\left((0, T) \times \mathbb{T}^{2} \times \zeta_{p, q}\right) \leq K_{p, q} \mu_{j}\left((0, T) \times \Omega \times \zeta_{p, q}\right) .
$$

\subsection{Conclusion of the proof of Proposition 4.1}

If the constant $K$ in the statement of the proposition is chosen so that, with $\delta$ in (4.7),

$$
\frac{K}{T}>\max \left(\frac{\operatorname{vol}\left(\mathbb{T}^{2}\right)}{\operatorname{vol}(\Omega)}, \frac{1}{\delta}, \underset{\sqrt{p^{2}+q^{2}} \leq N}{ } K_{p, q}\right),
$$

then, according to (4.6) and (4.7), we must have

$$
\mu\left((0, T) \times \mathbb{T}^{2} \times \mathbb{R}^{2}\right)<T,
$$

which contradicts (4.3) and completes the proof of Proposition 4.1.

\section{From smooth to rough potentials}

Proposition 4.1 was proved under the assumptions that $V_{j} \in C^{\infty}\left(\mathbb{T}^{2}\right)$ converge to $V \in$ $L^{2}\left(\mathbb{T}^{2}\right)$. To pass to $L^{2}$ potentials we will now use the results of $\S 2.2$.

\subsection{Classical observation estimate for smooth potentials}

The first proposition is the analogue of [8, Proposition 4.1] but with constants described by Proposition 4.1 .

Proposition 5.1. Suppose that $V_{j} \in C^{\infty}\left(\mathbb{T}^{2} ; \mathbb{R}\right)$ converge to $V$ in the $L^{2}\left(\mathbb{T}^{2}\right)$ topology. Then for any non-empty open subset $\Omega$ of $\mathbb{T}^{2}$ and $T>0$, there exists $C>0$ such that for any $j \in \mathbb{N}$ there exists $C_{j}$ such that for any $u_{0} \in L^{2}\left(\mathbb{T}^{2}\right)$, we have

$$
\left\|u_{0}\right\|_{L^{2}\left(\mathbb{T}^{2}\right)} \leq C\left\|e^{i t\left(\Delta-V_{j}\right)} u_{0}\right\|_{L^{2}((0, T) \times \Omega)}+C_{j}\left\|u_{0}\right\|_{H^{-2}\left(\mathbb{T}^{2}\right)} .
$$

Proof. To obtain the estimate (5.1) from Proposition 4.1, we apply pseudodifferential calculus in the time variable. This was already performed in [8], but since we need a precise dependence on the constants we recall the argument. Consider a $j$-dependent partition of unity

$$
\begin{aligned}
& 1=\varphi_{0, j}(r)^{2}+\sum_{k=1}^{\infty} \varphi_{k, j}(r)^{2}, \quad \varphi_{k, j}(r):=\varphi\left(R_{j}^{-k}|r|\right), \quad R>1, \\
& \varphi \in C_{0}^{\infty}\left(\left(R_{j}^{-1}, R_{j}\right) ;[0,1]\right), \quad\left(R_{j}^{-1}, R_{j}\right) \subset\left\{r ; \chi\left(r / \rho_{j}\right) \geq 1 / 2\right\},
\end{aligned}
$$


where $\chi$ and $\rho_{j}$ come from Proposition 4.1. Then, we decompose $u_{0}$ dyadically:

$$
\left\|u_{0}\right\|_{L^{2}}^{2}=\sum_{k=0}^{\infty}\left\|\varphi_{k, j}\left(P_{V_{j}}\right) u_{0}\right\|_{L^{2}}^{2}, \quad P_{V_{j}}:=-\Delta+V_{j} .
$$

Let $\psi \in C_{0}^{\infty}((0, T) ;[0,1])$ satisfy $\psi(t)>1 / 2$ for $T / 3<t<2 T / 3$. We first observe (using the time translation invariance of the Schrödinger equation) that in Proposition 4.1 we have actually proved that

$$
\left\|\Pi_{h, \rho_{j}, j} u_{0}\right\|_{L^{2}}^{2} \leq K \int_{\mathbb{R}} \psi(t)^{2}\left\|e^{-i t\left(-\Delta+V_{j}\right)} \Pi_{h, \rho_{j}, j} u_{0}\right\|_{L^{2}(\Omega)}^{2} d t, \quad 0<h<h_{0},
$$

which is the version we will use.

Taking $K_{j}$ large enough so that $R^{-K_{j}} \leq h_{0, j}$, where $h_{0, j}$ is as in Proposition 4.1, we apply (5.2) to the dyadic pieces:

$$
\begin{aligned}
\left\|u_{0}\right\|_{L^{2}}^{2} & =\sum_{k \in \mathbb{Z}}\left\|\varphi_{k, j}\left(P_{V_{j}}\right) u_{0}\right\|_{L^{2}}^{2} \\
& \leq \sum_{k=0}^{K_{j}}\left\|\varphi_{k, j}\left(P_{V_{j}}\right) u_{0}\right\|_{L^{2}}^{2}+C \sum_{k=K_{j}+1}^{\infty} \int_{0}^{T} \psi(t)^{2}\left\|\varphi_{k, j}\left(P_{V_{j}}\right) e^{-i t P_{V_{j}}} u_{0}\right\|_{L^{2}(\Omega)}^{2} d t \\
& =\sum_{k=0}^{K_{j}}\left\|\varphi_{k, j}\left(P_{V_{j}}\right) u_{0}\right\|_{L^{2}}^{2}+C \sum_{k=K_{j}+1}^{\infty} \int_{\mathbb{R}}\left\|\psi(t) \varphi_{k, j}\left(P_{V_{j}}\right) e^{-i t P_{V_{j}}} u_{0}\right\|_{L^{2}(\Omega)}^{2} d t .
\end{aligned}
$$

Using the equation we can replace $\varphi\left(P_{V_{j}}\right)$ by $\varphi\left(D_{t}\right)$, which means that we do not change the domain of $z$ integration. We need to consider the commutator of $\psi \in C_{0}^{\infty}((0, T))$ and $\varphi_{k, j}\left(D_{t}\right)=\varphi\left(R^{-j} D_{t}\right)$. If $\widetilde{\psi} \in C_{0}^{\infty}((0, T))$ is equal to 1 on supp $\psi$ then the semiclassical pseudodifferential calculus with $h=R_{j}^{-k}$ (see for instance [23, Chapter 4]) gives

$$
\psi(t) \varphi_{k, j}\left(D_{t}\right)=\psi(t) \varphi_{k, j}\left(D_{t}\right) \tilde{\psi}(t)+E_{j}\left(t, D_{t}\right), \quad \partial^{\alpha} E_{j}=\mathcal{O}\left(\langle t\rangle^{-N}\langle\tau\rangle^{-N} R_{j}^{-N k}\right),
$$

for all $N$ and uniformly in $k$.

The errors obtained from $E_{k}$ can be absorbed into the $\left\|u_{0}\right\|_{H^{-2}\left(\mathbb{T}^{2}\right)}$ term on the righthand side (with a constant depending on $j$ ). Hence we obtain

$$
\begin{aligned}
\left\|u_{0}\right\|_{L^{2}}^{2} & \leq C_{j}\left\|u_{0}\right\|_{H^{-2}\left(\mathbb{T}^{2}\right)}^{2}+C \sum_{k=0}^{\infty} \int_{0}^{T}\left\|\psi(t) \varphi_{k, j}\left(D_{t}\right) e^{-i t P_{V_{j}}} u_{0}\right\|_{L^{2}(\Omega)}^{2} d t \\
& \leq \widetilde{C}_{j}\left\|u_{0}\right\|_{H^{-2}\left(\mathbb{T}^{2}\right)}^{2}+K \sum_{k=0}^{\infty}\left\langle\varphi_{k, j}\left(D_{t}\right)^{2} \widetilde{\psi}(t) e^{-i t P_{V_{j}}} u_{0}, \widetilde{\psi}(t) e^{-i t P_{V_{j}}} u_{0}\right\rangle_{L^{2}\left(\mathbb{R}_{t} \times \Omega\right)} \\
& =\widetilde{C}_{j}\left\|u_{0}\right\|_{H^{-2}\left(\mathbb{T}^{2}\right)}^{2}+K \int_{\mathbb{R}}^{T}\left\|\widetilde{\psi}(t) e^{-i t P_{V}} u_{0}\right\|_{L^{2}(\Omega)}^{2} d t \\
& \leq \widetilde{C}_{j}\left\|u_{0}\right\|_{H^{-2}\left(\mathbb{T}^{2}\right)}^{2}+K \int_{0}^{T}\left\|e^{-i t P_{V}} u_{0}\right\|_{L^{2}(\Omega)}^{2} d t
\end{aligned}
$$

where the last inequality is the statement of the proposition. 


\subsection{Proof of Theorem 2}

We can now deduce Theorem 2 from Proposition 5.1. For that we consider a sequence $V_{j}$ of smooth potentials converging to $V$ in $L^{2}\left(\mathbb{T}^{2}\right)$ (to construct such a sequence, consider the Littlewood-Paley cut-off $V_{j}=\chi\left(2^{-2 j} \Delta\right) V$ with $\chi \in C_{0}^{\infty}(\mathbb{R})$ equal to 1 near 0$)$. We now have, according to Proposition 5.1,

$$
\left\|u_{0}\right\|_{L^{2}\left(\mathbb{T}^{2}\right)} \leq C\left\|e^{i t\left(\Delta-V_{j}\right)} u_{0}\right\|_{L^{2}((0, T) \times \Omega)}+D_{j}\left\|u_{0}\right\|_{H^{-2}\left(\mathbb{T}^{2}\right)} .
$$

On the other hand, according to (2.21), we have

$$
\left\|e^{i t\left(\Delta-V_{j}\right)} u_{0}\right\|_{L^{2}((0, T) \times \Omega)} \leq\left\|e^{i t(\Delta-V)} u_{0}\right\|_{L^{2}((0, T) \times \Omega)}+C\left\|V-V_{j}\right\|_{L^{2}\left(\mathbb{T}^{2}\right)}\left\|u_{0}\right\|_{L^{2}\left(\mathbb{T}_{x}^{2}\right)},
$$

hence, we deduce

$\left\|u_{0}\right\|_{L^{2}\left(\mathbb{T}^{2}\right)} \leq C\left\|e^{i t(\Delta-V)} u_{0}\right\|_{L^{2}((0, T) \times \Omega)}+C\left\|V-V_{j}\right\|_{L^{2}\left(\mathbb{T}^{2}\right)}\left\|u_{0}\right\|_{L^{2}\left(\mathbb{T}_{x}^{2}\right)}+D_{j}\left\|u_{0}\right\|_{H^{-2}\left(\mathbb{T}^{2}\right)}$, and consequently, taking $j$ large enough so that $C\left\|V-V_{j}\right\|_{L^{2}\left(\mathbb{T}^{2}\right)} \leq 1 / 2$, we conclude that

$$
\left\|u_{0}\right\|_{L^{2}\left(\mathbb{T}^{2}\right)} \leq 2 C\left\|e^{i t(\Delta-V)} u_{0}\right\|_{L^{2}((0, T) \times \Omega)}+2 D_{j}\left\|u_{0}\right\|_{H^{-2}\left(\mathbb{T}^{2}\right)} .
$$

It remains to eliminate the last term on the right-hand side. For this we use again the classical uniqueness-compactness argument of Bardos-Lebeau-Rauch [2] (see also [8, $\S 4]$ ) or the direct argument presented in the Appendix. The needed unique continuation result for $L^{2}$ potentials in $\mathbb{R}^{2}$ follows, as it did in $\S 2.1$, from the results of [21].

\section{Appendix A. A quantitative version of the uniqueness-compactness argument}

We present an abstract result which eliminates the low-frequency contributions in observability estimates.

Let $P$ be an unbounded self-adjoint operator on a Hilbert space $\mathcal{H}$. We assume that the spectrum of $P$ is discrete:

$$
P \varphi_{n}=\lambda_{n} \varphi_{n}, \quad \lambda_{1} \leq \lambda_{2} \leq \cdots, \quad \lambda_{n} \geq n^{\delta} / C_{0}, \quad \delta>0,
$$

where $\{\varphi\}_{n=1}^{\infty}$ form an orthonormal basis of $\mathcal{H}$.

We define $P$-based Sobolev spaces using the norms

$$
\|\varphi\|_{\mathcal{H}_{P}^{s}}^{2}:=\sum_{n=1}^{\infty}\left\langle\lambda_{n}\right\rangle^{2 s}\left|\left\langle\varphi, \varphi_{n}\right\rangle\right|^{2}
$$

The Schrödinger group for $P$ is formed by the following unitary operators on $\mathcal{H}$ :

$$
U(t) \varphi=\exp (-i t P) \varphi=\sum_{n=1}^{\infty}\left\langle\varphi, \varphi_{n}\right\rangle e^{-i t \lambda_{n}} \varphi_{n} .
$$

We have the following general result: 
Theorem 4. Suppose that $A: \mathcal{H} \rightarrow \mathcal{H}$ is a bounded operator with the property that for any $\lambda \in \mathbb{R}$ there exists a constant $C(\lambda)$ such that for $\varphi \in \mathcal{H}_{P}^{2}$,

$$
\|\varphi\|_{\mathcal{H}} \leq C(\lambda)\left(\|(P-\lambda) \varphi\|_{\mathcal{H}}+\|A \varphi\|_{\mathcal{H}}\right) .
$$

Suppose also that for some $\epsilon>0, T>0, C_{1}$ and $C_{2}$,

$$
\|\varphi\|_{\mathcal{H}}^{2} \leq C_{1} \int_{0}^{t}\|A U(s) \varphi\|_{\mathcal{H}}^{2} d s+C_{2}\|\varphi\|_{\mathcal{H}_{P}^{-\epsilon}}^{2}, \quad T / 4 \leq t \leq T .
$$

Then there exists an explicitly computable constant $K$ such that

$$
\|\varphi\|_{\mathcal{H}}^{2} \leq K \int_{0}^{T}\|A U(t) \varphi\|_{\mathcal{H}}^{2} d t
$$

Remarks. 1. We do not compute the constant explicitly but the construction in the proof certainly allows that.

2. In the applications in this paper,

$$
P=-\Delta+V, \quad \mathcal{H}=L^{2}\left(\mathbb{T}^{2}\right), \quad A=\mathbb{1}_{\Omega}, \quad \Omega \subset \mathbb{T}^{2} \text { open },
$$

or

$$
P=-\left(\partial_{x}+i k\right)^{2}+W, \quad \mathcal{H}=L^{2}\left(\mathbb{T}^{1}\right), \quad A=\mathbb{1}_{\omega}, \quad \omega \subset \mathbb{T}^{1} \text { open }
$$

Proof. We start by observing that (A.3) and the definition (A.1) imply that for $N>$ $\left(2 C_{2}\right)^{1 / \epsilon}$,

$$
\begin{gathered}
\|(I-\Pi) \varphi\|^{2} \leq 2 C_{1} \int_{0}^{t}\|A U(s)(I-\Pi) \varphi\|^{2} d s, \quad T / 4 \leq t \leq T, \\
\Pi \varphi:=\sum_{\lambda_{n} \leq N}\left\langle\varphi, \varphi_{n}\right\rangle \varphi_{n} .
\end{gathered}
$$

For reasons which will be explained below we will use this inequality for $t=T / 4$ and apply it with $\varphi$ replaced by $U(T / 2) \varphi$ :

$$
\|(I-\Pi) \varphi\|^{2} \leq 2 C_{1} \int_{T / 2}^{3 T / 4}\|A U(t)(I-\Pi) \varphi\|^{2} d t .
$$

We will show that the same estimate is true for $\Pi \varphi$. For that let $\mu_{1}<\cdots<\mu_{r_{1}}$ be the enumeration of $\left\{\lambda_{n}\right\}_{n=1}^{K_{1}}$ and define

$$
\psi_{r}:=\sum_{\lambda_{n}=\mu_{r}}\left\langle\varphi, \varphi_{n}\right\rangle \varphi_{n},
$$

so that

$$
U(t) \Pi \varphi=\sum_{n \leq K_{1}} e^{-i \lambda_{n} t}\left\langle\varphi, \varphi_{n}\right\rangle \varphi_{n}=\sum_{r=1}^{r_{1}} e^{i \mu_{r} t} \psi_{r}
$$


Since $\left(P-\mu_{r}\right) \psi_{r}=0$, we can apply (A.2) to obtain

$$
\left\|\psi_{r}\right\| \leq K_{2}\left\|A \psi_{r}\right\|, \quad K_{2}=\max _{n \leq K_{1}} C\left(\lambda_{n}\right)
$$

The functions $t \mapsto e^{i \mu_{r} t}, r=1, \ldots, r_{1}$, are linearly independent and there exists a constant $K_{3}=K_{3}\left(\mu_{1}, \ldots, \mu_{r_{1}}, T\right)$ such that for any $f_{1}, \ldots, f_{r_{1}} \in \mathcal{H}$,

$$
\int_{T / 2}^{3 T / 4}\left\|\sum_{r=1}^{r_{1}} e^{i \mu_{r} t} f_{r}\right\|^{2} d t \geq K_{3} \sum_{r=1}^{r_{1}}\left\|f_{r}\right\|^{2},
$$

as both sides provide equivalent norms on $\times_{r=1}^{r_{1}} \mathcal{H}$.

Applying (A.8) with $f_{r}=A \psi_{r}$ and (A.7) gives

$$
\begin{aligned}
\|A U(t) \Pi \varphi\|_{L^{2}((T / 2,3 T / 4) ; \mathcal{H})}^{2} & =\int_{T / 2}^{3 T / 4}\left\|\sum_{r=1}^{r_{1}} A \psi_{r} e^{i \mu_{r} t}\right\|^{2} d t \geq K_{2} \sum_{r=1}^{r_{1}}\left\|A \psi_{r}\right\|^{2} \\
& \geq K_{2} K_{3} \sum_{r=1}^{r_{1}}\left\|\psi_{r}\right\|^{2}=K_{2} K_{3}\|\Pi \varphi\| .
\end{aligned}
$$
if

The combination of (A.6) and (A.9) does not yet provide the estimate (A.4). However,

$$
\Pi_{M} \varphi:=\sum_{\lambda_{n} \leq M}\left\langle\varphi, \varphi_{n}\right\rangle \varphi_{n}
$$

then for $M$ sufficiently large we have

$$
\begin{aligned}
\left\|A U(t)\left(I-\Pi_{M}+\Pi\right) \varphi\right\|_{L^{2}((0, T) ; \mathcal{H})}^{2} & \\
& \geq K_{2}^{2} K_{3}^{2}\|\Pi \varphi\|^{2}+\left(1 / 4 C_{1}^{2}\right)\left\|\left(I-\Pi_{M}\right) \varphi\right\|^{2}-K_{4} M^{-1}\|\varphi\|^{2},
\end{aligned}
$$

where $K_{4}$ will be defined below. In fact, if we choose $\eta \in C_{0}^{\infty}((0, T))$ equal to 1 on $[T / 2,3 T / 4]$, then the left-hand side in (A.10) is estimated from below by

$$
\begin{aligned}
\int \| A U(t)(I & \left.-\Pi_{M}+\Pi\right) \varphi\left\|^{2} \eta(t) d t=\int\right\| A U(t)\left(I-\Pi_{M}\right) \varphi \|^{2} \eta(t) d t \\
& +\int\|A U(t) \Pi \varphi\|^{2} \eta(t) d t-2 \operatorname{Re} \int\left\langle A U(t)\left(I-\Pi_{M}\right) \varphi, A U(t) \Pi \varphi\right\rangle \eta(t) d t .
\end{aligned}
$$

We can apply (A.5) and (A.9) to estimate the first two terms from below. Since

$$
\begin{aligned}
2 \operatorname{Re} \int\langle A U(t) & \left.\left(I-\Pi_{M}\right) \varphi, A U(t) \Pi \varphi\right\rangle \eta(t) d t \\
& =2 \operatorname{Re} \sum_{\lambda_{n}<N} \sum_{\lambda_{m}>M}\left\langle\varphi, \varphi_{n}\right\rangle\left\langle\varphi_{m}, \varphi\right\rangle\left\langle A \varphi_{n}, A \varphi_{m}\right\rangle \int e^{i\left(\lambda_{n}-\lambda_{m}\right) t} \eta(t) d t \\
& \leq C_{P}\|A\|^{2} \sum_{\lambda_{n}<N} \sum_{\lambda_{m}>M}\left|\lambda_{n}-\lambda_{m}\right|^{-P}\|\varphi\|^{2} \leq K_{4} M^{-1}\|\varphi\|^{2}
\end{aligned}
$$

if we choose $P$ sufficiently large, we obtain (A.10). 
We now have to deal with the remaining eigenfunctions corresponding to $N \leq \lambda_{n}<M$. Let $\mu_{r_{1}+1}<\cdots<\mu_{r_{2}}$ be the enumeration of these eigenvalues. Put

$$
\tau=\frac{T}{10 r_{2}}
$$

The Vandermonde matrix $\left(e^{i \mu_{r} p \tau}\right)_{1 \leq r \leq r_{2}, 1 \leq p \leq r_{2}}$ is non-singular and hence we can find scalars $\sigma_{p}$ with $\max \left|\sigma_{p}\right|=1$ satisfying

$$
\sum_{p=1}^{r_{2}} \sigma_{p} e^{i \mu_{r} p \tau}=0 \quad \text { for } r \leq r_{1}, \quad\left|\sum_{p=1}^{r_{2}} \sigma_{p} e^{i \mu_{r} p \tau}\right| \geq K_{5} \quad \text { for } r_{1}<r \leq r_{2},
$$

with a constant $K_{5}=K_{5}\left(\mu_{1}, \ldots, \mu_{r_{2}}, T\right)$. (Note the implicit dependence on $M$.)

If we define

$$
\tilde{\varphi}=\sum_{\lambda_{n}>N}\left(\sum_{r=1}^{r_{2}} \sigma_{p} e^{i \lambda_{n} p \tau}\right)\left\langle\varphi, \varphi_{n}\right\rangle \varphi_{n}
$$

then

$$
(I-\Pi) \tilde{\varphi}=\tilde{\varphi} \quad \text { and } \quad U(t) \tilde{\varphi}=\sum_{r=1}^{r_{2}} \sigma_{p} U(t+p \tau) \varphi .
$$

Applying (A.5), (A.12) and the definition (A.13) gives

$$
\begin{aligned}
4 C_{1}^{2}\|A U(t) \tilde{\varphi}\|_{L^{2}((T / 2,3 T / 4) ; \mathcal{H})}^{2} & \geq\|\tilde{\varphi}\|^{2} \geq \sum_{N \leq \lambda_{n}<M}\left|\sum_{r=1}^{r_{2}} \sigma_{p} e^{i \lambda_{n} p \tau}\right|^{2}\left|\left\langle\varphi, \varphi_{n}\right\rangle\right|^{2} \\
& \geq K_{5}^{2}\left\|\left(\Pi_{M}-\Pi\right) \varphi\right\|^{2} .
\end{aligned}
$$

The choice of $\tau$ in (A.11) and (A.14) show that

$$
\|A U(t) \varphi\| \geq \frac{K_{5}}{2 C_{1} r_{2}}\left\|\left(\Pi_{M}-\Pi\right) \varphi\right\|^{2} .
$$

This gives

$$
\begin{aligned}
\left\|A U(t)\left(I-\Pi_{M}+\Pi\right) \varphi\right\|_{L^{2}((0, T) ; \mathcal{H})} & \leq\|A U(t) \varphi\|_{L^{2}((0, T) ; \mathcal{H})}+\sqrt{T}\left\|\left(\Pi_{M}-\Pi\right) \varphi\right\| \\
& \leq\left(1+\frac{2 \sqrt{T} r_{2} C_{1}}{K_{5}}\right)\|A U(t) \varphi\|_{L^{2}((0, T) ; \mathcal{H})},
\end{aligned}
$$

which combined with (A.10) and (A.15) produces

$$
\begin{aligned}
\left(1+\frac{2(\sqrt{T}+1) r_{2} C_{1}}{K_{5}}\right)\|A U(t) \varphi\|_{L^{2}((0, T) ; \mathcal{H}) \geq} & K_{2} K_{3}\|\Pi \varphi\|+\left(1 / 2 C_{1}\right)\left\|\left(I-\Pi_{M}\right) \varphi\right\| \\
& +\left\|\left(\Pi_{M}-\Pi\right) \varphi\right\|-\sqrt{K_{4} / M}\|\varphi\|^{2} \\
\geq & \left(K_{6}-\sqrt{K_{4} / M}\right)\|\varphi\| .
\end{aligned}
$$

A $K_{6}$ and $K_{4}$ are independent of $M$, we obtain (A.4) by choosing $M$ large enough. 


\section{Appendix B. Proof of Lemma 2.5}

This is a purely geometric result which does not involve integer points. It is a consequence of the fact that the circle is curved but we prove it by explicit calculations.

We start with the case where $\gamma=1$ (recall that in Lemma 2.5 the modulus is defined by $\left.\left|\left(x_{1}, x_{2}\right)\right|^{2}=x_{1}^{2}+\gamma x_{2}^{2}\right)$. We perform a change of variables $x \mapsto x h$, and denote $\epsilon=\kappa^{2} h^{2}$. We are reduced to proving that for

$$
\mathcal{B}_{\epsilon, \alpha}=\{z \in \mathbb{C} ; \operatorname{Re} z \geq 0, \operatorname{Im} z \geq 0,|| z|-1| \leq \epsilon, \arg (z) \in[\alpha \sqrt{\epsilon},(\alpha+1) \sqrt{\epsilon})\},
$$

we have

Lemma B.1. There exist $\epsilon_{0}>0$ and $Q>0$ such that for any $0<\epsilon \leq \epsilon_{0}$, we have

$$
\begin{aligned}
& \forall \alpha_{j} \in\left\{0,1, \ldots, N_{\epsilon}\right\}, j=1, \ldots 4, N_{\epsilon}:=[\pi / 2 \sqrt{\epsilon}] \\
& \left(\mathcal{B}_{\epsilon, \alpha_{1}}+\mathcal{B}_{\epsilon, \alpha_{2}}\right) \cap\left(\mathcal{B}_{\epsilon, \alpha_{3}}+\mathcal{B}_{\epsilon, \alpha_{4}}\right) \neq \emptyset \\
& \quad \Rightarrow\left|\alpha_{1}-\alpha_{3}\right|+\left|\alpha_{2}-\alpha_{4}\right| \leq Q \text { or }\left|\alpha_{1}-\alpha_{4}\right|+\left|\alpha_{2}-\alpha_{3}\right| \leq Q .
\end{aligned}
$$

Proof. We first observe that it is enough to prove the lemma with the condition ||$z|-1|$ $<\epsilon$ replaced by $0 \leq|z|-1 \leq \epsilon$ in the definition of $\mathcal{B}_{\epsilon, \alpha}: 0 \leq 1-|z| \leq \epsilon$ is the same as $0 \leq|z| /(1-\epsilon)-1 \leq \epsilon /(1-\epsilon)$.

Let $z_{j}=\rho_{j} e^{i \theta_{j}} \in \mathcal{B}_{\epsilon, \alpha_{j}}, 1 \leq j \leq 4$, be such that $z_{1}+z_{2}=z_{3}+z_{4}$. By possibly exchanging $z_{1}$ and $z_{2}$ we can assume $\theta_{1} \geq \theta_{2}$ and similarly that $\theta_{3} \geq \theta_{4}$. In particular,

$$
\left(\theta_{1}-\theta_{2}\right) / 2 \in[0, \pi / 4], \quad\left(\theta_{3}-\theta_{4}\right) / 2 \in[0, \pi / 4] .
$$

Since $\rho_{j} \in[1,1+\epsilon]$, we have

$$
\left|e^{i \theta_{1}}+e^{i \theta_{2}}-e^{i \theta_{3}}-e^{i \theta_{4}}\right| \leq 4 \epsilon,
$$

which is the same as

$$
\left|e^{i / 2\left(\theta_{1}+\theta_{2}\right)} \cos \left(\frac{\theta_{1}-\theta_{2}}{2}\right)-e^{i / 2\left(\theta_{3}+\theta_{4}\right)} \cos \left(\frac{\theta_{3}-\theta_{4}}{2}\right)\right| \leq 2 \epsilon .
$$

On the other hand,

$$
\begin{aligned}
\mid e^{\frac{i}{2}\left(\theta_{1}+\theta_{2}\right)} & \cos \left(\frac{\theta_{1}-\theta_{2}}{2}\right)-e^{\frac{i}{2}\left(\theta_{3}+\theta_{4}\right)} \cos \left(\frac{\theta_{3}-\theta_{4}}{2}\right) \mid \\
= & \left|e^{i / 2\left(\theta_{1}+\theta_{2}-\theta_{3}-\theta_{4}\right)} \cos \left(\frac{\theta_{1}-\theta_{2}}{2}\right)-\cos \left(\frac{\theta_{3}-\theta_{4}}{2}\right)\right| \geq\left|\sin \left(\frac{\theta_{1}+\theta_{2}-\theta_{3}-\theta_{4}}{2}\right) \cos \left(\frac{\theta_{1}-\theta_{2}}{2}\right)\right| .
\end{aligned}
$$

Since (B.3) implies that $\cos \left(\frac{\theta_{1}-\theta_{2}}{2}\right) \geq 1 / \sqrt{2}$, we deduce from (B.4) that

$$
\left|\sin \left(\frac{\theta_{1}+\theta_{2}-\theta_{3}-\theta_{4}}{2}\right)\right| \leq 2 \sqrt{2} \epsilon \text {. }
$$

We also have $\left(\theta_{1}+\theta_{2}-\theta_{3}-\theta_{4}\right) / 2 \in[-\pi / 2, \pi / 2]$ and as $|\sin \theta| \geq 2|\theta| / \pi$ for $-\pi / 2 \leq$ $\theta \leq \pi / 2$, we conclude that

$$
\left|\frac{\theta_{1}+\theta_{2}-\theta_{3}-\theta_{4}}{2}\right| \leq \pi \sqrt{2} \epsilon
$$


We assumed that $z_{j}=\rho_{j} e^{i \theta_{j}} \in \mathcal{B}_{\epsilon, \alpha_{j}}$ and that means that $0 \leq \theta_{j}-\sqrt{\epsilon} \alpha_{j}<\sqrt{\epsilon}$. Hence (B.5) gives

$$
\left|\alpha_{1}+\alpha_{2}-\alpha_{3}-\alpha_{4}\right| \leq C \sqrt{\epsilon}+2 \leq 3,
$$

provided that $\epsilon>0$ small enough.

Going back to (B.3) and (B.4) we get, with $p=\frac{\theta_{1}-\theta_{2}}{2}, q=\frac{\theta_{3}-\theta_{4}}{2}$,

$$
|\cos p-\cos q|=2\left|\sin \left(\frac{p+q}{2}\right) \sin \left(\frac{p-q}{2}\right)\right| \leq 2 \epsilon .
$$

As, $p, q \in[0, \pi / 4]$ we get

$$
\left|\frac{p+q}{2} \frac{p-q}{2}\right| \leq \frac{\pi^{2}}{4} \epsilon .
$$

This is the same as (recall that $0 \leq \theta_{1}-\theta_{2}, 0 \leq \theta_{3}-\theta_{4}$ )

$$
\left(\left|\theta_{1}-\theta_{2}\right|-\left|\theta_{3}-\theta_{4}\right|\right)\left(\left|\theta_{1}-\theta_{2}\right|+\left|\theta_{3}-\theta_{4}\right|\right) \leq 4 \pi^{2} \epsilon,
$$

and this gives

$$
\left|\left(\theta_{1}-\theta_{2}\right)-\left(\theta_{3}-\theta_{4}\right)\right| \leq\left(\left(\left|\theta_{1}-\theta_{2}\right|-\left|\theta_{3}-\theta_{4}\right|\right)\left(\left|\theta_{1}-\theta_{2}\right|+\left|\theta_{3}-\theta_{4}\right|\right)\right)^{1 / 2} \leq 2 \pi \sqrt{\epsilon} .
$$

Using again the fact that $0 \leq \theta_{j}-\sqrt{\epsilon} \alpha_{j}<\sqrt{\epsilon}$, this gives

$$
\left|\left(\alpha_{1}-\alpha_{2}\right)-\left(\alpha_{3}-\alpha_{4}\right)\right| \leq 2 \pi+2 .
$$

Finally, from (B.6) and (B.10) we obtain

$$
\left|\alpha_{1}-\alpha_{3}\right| \leq \pi+5 / 2, \quad\left|\alpha_{2}-\alpha_{4}\right| \leq \pi+5 / 2,
$$

which proves Lemma 2.5 in the case $\gamma=1$ (notice that here only the first term in the alternative is possible which follows from the assumption $\theta_{1} \geq \theta_{2}, \theta_{3} \geq \theta_{4}$ ). The general case follows by applying the transformation $\left(x_{1}, x_{2}\right) \in \mathbb{R}^{2} \mapsto\left(x_{1}, \sqrt{\gamma} x_{2}\right) \in \mathbb{R}^{2}$.

Acknowledgments. This research was partially supported by NSF grants DMS-0808042, DMS0835373 (JB) and DMS-1201417 (MZ).

\section{References}

[1] Anantharaman, N., Macià, F.: Semiclassical measures for the Schrödinger equation on the torus. arXiv:1005.0296.

[2] Bardos, C., Lebeau, G., Rauch, J.: Sharp sufficient conditions for the observation, control, and stabilization of waves from the boundary. SIAM J. Control Optim. 30, 1024-1065 (1992) Zbl 0786.93009 MR 1178650

[3] Bourgain, J., Shao, P., Sogge, C. D., Yao, X.: On $L^{p}$-resolvent estimates and the density of eigenvalues for compact Riemannian manifolds. arXiv:1204.3927 (2012)

[4] Burq, N.: Semi-classical measures for inhomogeneous Schrödinger equations on tori. Anal. PDE, to appear; arXiv:1209.3739

[5] Burq, N., Gérard, P., Tzvetkov, N.: An instability property of the nonlinear Schrödinger equation on $S^{d}$. Math. Res. Lett. 9, 323-335 (2002) Zbl 1003.35113 MR 1909648 
[6] Burq, N., Zworski, M.: Geometric control in the presence of a black box. J. Amer. Math. Soc. 17, 443-471 (2004) Zbl 1050.35058 MR 2051618

[7] Burq, N., Zworski, M.: Bouncing ball modes and quantum chaos. SIAM Rev. 47, 43-49 (2005) Zbl 1072.81022 MR 2149100

[8] Burq, N., Zworski, M.: Control for Schrödinger equations on tori. Math. Res. Lett. 19, 309324 (2012) MR 2955763

[9] Córdoba, A.: Geometric Fourier analysis. Ann. Inst. Fourier (Grenoble) 32, no. 3, 215-226 (1982) Zbl 0488.42027 MR 0688026

[10] Dimassi, M., Sjöstrand, J.: Spectral Asymptotics in the Semiclassical Limit. London Math. Soc. Lecture Note Ser. 268, Cambridge Univ. Press, Cambridge (1999) Zbl 0926.35002 MR 1735654

[11] Dos Santos, D., Kenig, C., Salo, M.: On $L^{p}$-resolvent estimates for Laplace-Beltrami operators on compact manifolds. Forum Math., to appear; arXiv:1112.3216

[12] Haraux, A.: Séries lacunaires et contrôle semi-interne des vibrations d'une plaque rectangulaire. J. Math. Pures Appl. 68, 457-465 (1989) Zbl 0685.93039 MR 1046761

[13] Jaffard, S.: Contrôle interne exact des vibrations d'une plaque rectangulaire. Portugal. Math. 47, 423-429 (1990) Zbl 0718.49026 MR 1090480

[14] Jerison, D., Kenig, C. E.: Unique continuation and absence of positive eigenvalues for Schrödinger operators. Ann. of Math. 121, 463-488 (1985) Zbl 0593.35119 MR 0794370

[15] Kahane, J.-P.: Pseudo-périodicité et séries de Fourier lacunaires. Ann. Sci. École Norm. Sup. 79, 93-150 (1962) Zbl 0105.28601 MR 0154060

[16] Komornik, V.: On the exact internal controllability of a Petrowsky system. J. Math. Pures Appl. 71, 331-342 (1992) Zbl 0889.34055 MR 1176015

[17] Kahane, J.-P.: Pseudo-périodicité et séries de Fourier lacunaires. Ann. Sci. École Norm. Sup. 79, 93-150 (1962) Zbl 0105.28601 MR 0154060

[18] Lebeau, G.: Contrôle de l'équation de Schrödinger. J. Math. Pures Appl. 71, 267-291 (1992) Zbl 0838.35013 MR 1172452

[19] Lions, J.-L.: Contrôlabilité exacte, perturbation et stabilisation des systèmes distribués. Tome 2, Recherches Math. Appl. 9, Masson (1988) Zbl 0653.93003 MR 0963060

[20] Miller, L.: Controllability cost of conservative systems: resolvent condition and transmutation. J. Funct. Anal. 218, 425-444 (2005) Zbl 1122.93011 MR 2108119

[21] Schechter, M., Simon, B.: Unique continuation for Schrödinger operators with unbounded potential. J. Math. Anal. Appl. 77, 482-492 (1980) Zbl 0458.35024 MR 0593229

[22] Sogge, C.: Concerning the $L^{p}$ norm of spectral clusters for second order elliptic operators on compact manifolds. J. Funct. Anal. 77, 123-138 (1988) Zbl 0641.46011 MR 0930395

[23] Zworski, M.: Semiclassical Analysis. Grad. Stud. Math. 138, Amer. Math. Soc (2012) Zbl 1252.58001 MR 2952218 